\title{
Improved Prediction of Properties of $\pi$-Conjugated Oligomers with Range-Separated Hybrid Density Functionals
}

\author{
Ulrike Salzner* and Aykut Aydin \\ Department of Chemistry, Bilkent University, 06800 Bilkent, Ankara, Turkey
}

ABSTRACT: Range-separated hybrid functionals along with global hybrids and pure density functionals have been employed to calculate geometries, ionization energies (IP)s, electron affinities (EA)s, and excitation energies of neutral and oxidized polyenes, thiophene, and furan oligomers. Long-range correction with $100 \% \mathrm{HF}$ exchange solves the problem of density functional theory with incorrect chain length dependence of IPs and energy gaps. There is a possibility of overcorrection, if the short-range part of the functional with no or low HF exchange is too small. The wB97XD functional with $22 \%$ of HF exchange in the short-range and a range-separation parameter of 0.2 seems to be just right for conjugated systems at all chain lengths. The wB97XD functional additionally produces negative orbital energies in very good agreement with IPs and EAs. With correct orbital energies, band gaps correspond to transport gaps $\left(E_{\mathrm{t}}\right)$ and not to optical gaps $\left(E_{\mathrm{g}}\right) . E_{\mathrm{t}}$ is much larger than $E_{\mathrm{g}}$ in the gas phase, but the difference is significantly smaller in the solid state. The accuracy of the negative orbital energies is good down to about $30 \mathrm{eV}$ so that valence and innervalence PE spectra can be modeled. wB97XD is therefore suitable for calculating band structures of conjugated polymers employing orbital energies.

\section{INTRODUCTION}

Crucial properties for theoretical research on conducting organic polymers (COPs) are ionization potentials (IP)s, electron affinities (EA)s, and excitation energies of neutral and charged species. These properties relate to experimental observables and determine ease of doping, stability in neutral and doped states, optical band gap $\left(E_{\mathrm{g}}\right)$, and conductivity. Instead of calculating states, band structure calculations are often used, which means that orbital energies and orbital energy differences are used as approximations for energies and densities of states. Therefore, an adequate theoretical level should correctly predict IPs and EAs, and both should match the corresponding negative orbital energies. To evaluate the density of states, lower and higher lying orbital energies have to match states, too. Because all of the properties depend on geometries, i.e., bond length alternation (BLA) in neutral systems and defect sizes in ions, geometries have to be predicted accurately.

In recent years, DFT has gradually replaced semiempirical methods, ${ }^{1-4}$ Hartree-Fock (HF) theory, ${ }^{5-7}$ and perturbation theory ${ }^{8,9}$ in research on COPs because it includes electronic interactions self-consistently, ${ }^{10}$ accounts for electron correlation, ${ }^{11}$ and avoids spin-contamination in open-shell systems. ${ }^{12,13}$ Despite many successes of DFT, a couple of vexing problems remain, however. Foremost, there is the incorrect chain length dependence of $\mathrm{IPs}^{14}$ and excitation energies ${ }^{15}$ of neutral systems, which leads to underestimation of polymer properties no matter whether periodic boundary conditions or extrapolation methods are applied. Another important issue is the mismatch between the negative energy of the highest occupied molecular orbital $\left(-\varepsilon_{\mathrm{HOMO}}\right)$ and $\mathrm{IP}_{\triangle \mathrm{SCF}}\left(\mathrm{E}_{\mathrm{cat}}-\mathrm{E}_{\text {neutral }}\right)$ as well as the experimental IP. $-\varepsilon_{\mathrm{HOMO}}$ should be identical with the $\mathrm{IP}_{\triangle \mathrm{SCF}}$ with the exact exchangecorrelation functional, ${ }^{16,17}$ but it differs by a couple electronvolts with most approximate functionals. ${ }^{18-21}$ Finally, there is controversy about geometries, as pure DFT underestimates bond length alternation, ${ }^{22,23}$ predicts larger defect sizes than semiempirical methods, ${ }^{24}$ and does not confirm the existence of bound bipolarons in doped systems. ${ }^{25-27}$

Some of the problems of DFT with extended $\pi$ systems can be ameliorated by the inclusion of HF exchange. ${ }^{18,23,28}$ It was shown, for instance, that bond length alternation increases to reasonable values with hybrid functionals, ${ }^{23}$ that defect sizes predicted with hybrid functionals agree with those at the MP2 level, ${ }^{12}$ that $\mathrm{HOMO}-\mathrm{LUMO}$ gaps $\left(E_{\mathrm{H}-\mathrm{L}}\right)$ can be adjusted with HF exchange to reproduce $E_{\mathrm{g}}, 23,28$ and that orbital energy differences agree better with differences between states. ${ }^{29}$ Nonetheless, no global hybrid functional is able to predict the correct chain length dependence of IPs, ${ }^{14} E_{\mathrm{g}}$ 's, $^{15}$ or $E_{\mathrm{H}-\mathrm{L}}$ 's. ${ }^{30}$ These errors are a consequence of the self-interaction error (SIE) that arises in DFT because exchange is approximated.

The SIE is now addressed with range-separated density functionals $^{31,32}$ that eliminate the SIE by using $100 \% \mathrm{HF}$ exchange for the long-range part of the exchange-correlation functional. $^{32-43}$ Therefore, SIE-free functionals with the correct long-range behavior of the exchange-correlation potential hold promise for improving the accuracy of theoretical results on extended $\pi$ systems. It is the goal of this investigation to test a selection of new density functionals with respect to IPs, EAs, fundamental gaps $\left(E_{\mathrm{t}}\right)$ and $E_{\mathrm{g}}$ 's, bipolaron binding energies, and excitation energies of doped $\pi$ systems. Guided by the availability of experimental data, polyenes, thiophene, and furan oligomers were chosen as representative systems.

\section{METHODS}

Structures of oligomers were optimized with a range of density functionals, as summarized below. If not stated otherwise, the

Received: $\quad$ May 21, 2011

Published: July 06, 2011 


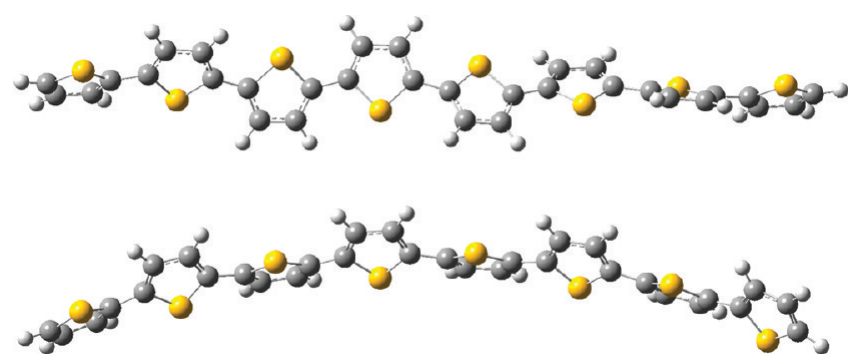

Figure 1. 8T with same sign dihedral angles (top) and alternating sign dihedral angles (bottom).

6-311G* basis set was employed. Thiophene and furan oligomers of different size are designated $n \mathrm{~T}$ and $n \mathrm{~F}$, respectively, $n$ being the number of rings. Polyenes ${ }^{44}$ and furanes ${ }^{45}$ are planar. Because bithiophene is nonplanar in the gas phase, ${ }^{46}$ thiophene oligomers were allowed to deviate from planarity. There are two main structural alternatives: one with all interring dihedral angles having the same sign and one with alternating signs (see Figure 1). The latter are slightly lower in energy and have about $0.1 \mathrm{eV}$ smaller excitation energies. This conformation leads to bending and may not be adopted in a crystal. The lower energy forms were used in the following because they are the lower energy structures in the gas phase and probably in solution. In all cases, planarization energies and influences on other properties due to bending are relatively small. Cation energies were obtained on the structures of the neutral forms to evaluate vertical IPs and with optimized geometries to evaluate UV spectra upon doping. Vertical IPs were calculated with the $\triangle$ SCF method as the difference between ground state energies of cation and neutral species and are abbreviated as $\mathrm{IP}_{\triangle \mathrm{SCF}}$.

Upon structure optimization, cations and anions become planar and straight with pure DFT and with global hybrids. With a range-separated hybrid functional, planarization occurs over five rings, but the chain ends remain slightly twisted, as shown in Figure 2 for $19 \mathrm{~T}^{+}$at the wB97XD/6-31G* level (description, see below). Excitation energies are calculated with time-dependent density functional theory. Most calculations were performed with Gaussian 09. ${ }^{47}$ Calculations with BNL were done with QChem. $^{48}$

The density functionals are grouped into pure local density functionals, gradient corrected (nonlocal) functionals (GGA), meta-GGAs that depend explicitly on the kinetic energy density, ${ }^{42,49-51}$ global hybrid functionals that use a constant amount of exact (HF) exchange, ${ }^{42}$ and range-separated functionals $^{32}$ that use different amounts of DFT and HF exchange for short and long ranges. For comparison, HF theory was included as well. The following density functionals were used:

Local Functionals. SVWN: Slater exchange ${ }^{52}$ and correlation functional of Vosko, Wilk, and Nussair, ${ }^{53}$ identical to local spin density approximation (LSDA). SVWN5: same as SVWN with correlation functional $\mathrm{V}$ from ref 53.

Gradient Corrected (GGA) Functionals. BLYP: gradient corrected functional with Becke exchange ${ }^{54}$ and correlation functional of Lee, Yang, and Parr. ${ }^{55}$ BP86: gradient corrected functional with Becke exchange ${ }^{54}$ and correlation functional of Perdew. ${ }^{56}$ PBEPBE: Perdew, Burke, and Ernzerhof ${ }^{57,58}$ exchange and Perdew, Burke, and Ernzerhof correlation functionals. ${ }^{57,58}$ OLYP: OPTX modification by Handy ${ }^{59}$ of Becke exchange ${ }^{54}$ and Lee, Yang, and Parr correlation functional. $^{55}$
Meta-GGA (Containing Explicit Dependence on the Kinetic Energy Density). ${ }^{42,49-51}$ TPSSTPSS: Exchange and correlation functional of Tao, Perdew, Staroverov, and Scuseria. ${ }^{50}$ Global GGA Hybrids (Constant Amount of HF Exchange). B3LYP: Becke's three-parameter hybrid functional with $20 \%$ HF exchange ${ }^{60}$ and correlation functional of Lee, Yang, and Parr. ${ }^{55}$ B3P86: Becke's three-parameter hybrid functional with $20 \%$ HF exchange ${ }^{60}$ with the correlation functional of Perdew. ${ }^{56} \mathrm{~B} 3 \mathrm{P} 86-30 \%$ : same as $\mathrm{B} 3 \mathrm{P} 86$, but HF exchange is increased to $30 \%$. PBE1PBE: also known as PBE0, hybrid version (25\% exchange and $75 \%$ correlation weighting) by Adamo and Barone ${ }^{61}$ of Perdew, Burke, and Ernzerhof ${ }^{57,58}$ exchange and Perdew, Burke, and Ernzerhof correlation functionals. ${ }^{57,58}$

Global meta-GGA Hybrids (Constant Amount of HF Exchange). M06: meta hybrid functional with $27 \%$ HF exchange. M06HF: $100 \%$ HF exchange.

Range Separated Hybrid Functionals without Short-Range HF Exchange. All local, GGA, and meta-GGA functionals listed above were also used in their long-range corrected version. For the long-range correction (LC) Hirao et al.'s range separation scheme $^{32}$ without short-range HF exchange and with a rangeseparation parameter $\gamma=0.4$ was employed. LC-wPBE: longrange corrected ${ }^{35,42,49,51}$ Perdew, Burke, and Ernzerhof ${ }^{57,38}$ functional. wB97: range-separated version ${ }^{62}$ of Becke's 97 functional $\gamma=0.4$. $^{63}$

Range Separated Hybrid Functionals with Short-Range HF Exchange. CAM-B3LYP: Coulomb attenuated version of B3LYP by Yanai et al., ${ }^{64}$ includes 19\% HF exchange at short range and $65 \% \mathrm{HF}$ exchange at long range, $\gamma=0.33$. wB97X: same as wB97 but containing $16 \%$ short-range HF exchange and $\gamma=0.3{ }^{62}$ wB97XD: same as above with additional dispersion correction, containing $22 \%$ short-range HF exchange and $\gamma=0.2$. $^{65}$

Range Separated Hybrid Functional with $\gamma$ Tuning. BNL: Baer-Neuhauser-Livshits ${ }^{37,43}$ using LDA exchange, the $\mathrm{LYP}^{55}$ correlation functional subtracting a small part of the Savin exchange. ${ }^{43} \gamma$ Tuning means that the range-separation parameter is adjusted to reproduce $\mathrm{IP}_{\Delta \mathrm{SCF}}=-\varepsilon_{\mathrm{HOMO}}$. Decreasing the value of $\gamma$ increases the extension of the short-range part. $\gamma=0$ reduces range-separated functionals to pure DFT or global hybrid functionals with constant HF exchange equal to that of the basic functional.

\section{RESULTS}

Degree of Planarity of Thiophene Oligomers. $6 \mathrm{~T}^{66}$ and $8 \mathrm{~T}^{67}$ have been crystallized, and their structural parameters have been determined. Although $2 \mathrm{~T}$ is nonplanar in the gas phase with an interring angle of $148^{\circ}, 46$ T and $8 \mathrm{~T}$ are planar in the crystal. We are not aware of gas-phase structures for $6 \mathrm{~T}$ and $8 \mathrm{~T}$. Theoretical results indicate that the interring dihedral angle in $6 \mathrm{~T}$ is larger than in $2 \mathrm{~T}$ but that $6 \mathrm{~T}$ is slightly nonplanar in the gas phase. For instance, the interring angles are $138.6^{\circ}(2 \mathrm{~T})$ and $145.7^{\circ}(6 \mathrm{~T})$ at the MP2/6-311G* level; the energy for planarization of $6 \mathrm{~T}$ is $1.4 \mathrm{kcal} / \mathrm{mol}$. The agreement with the experiment for $2 \mathrm{~T}$ is best with LC-BLYP, $147.0^{\circ}$; CAM-B3LYP, $147.0^{\circ}$; and B3P86-30\%/ $6-311 \mathrm{G}^{*}, 149.9^{\circ}$. Planarity increases more from $2 \mathrm{~T}$ to $6 \mathrm{~T}$ with global hybrids than with range-separated functionals to $150.6^{\circ}$ at LC-BLYP, $152.2^{\circ}$ at CAM-B3LYP, and $156.9^{\circ}$ at B3P86-30\%. In general, more HF exchange leads to stronger nonplanarity. 


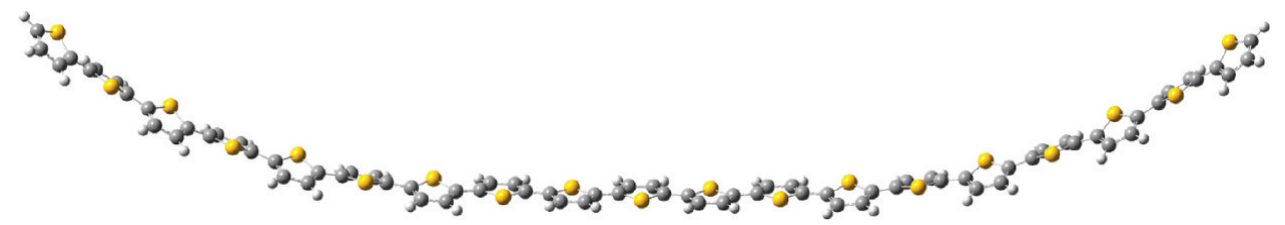

Figure 2. $19 \mathrm{~T}^{+}$optimized at $\mathrm{wB} 97 \mathrm{XD} / 6-31 \mathrm{G}^{*}$.

Table 1. Theoretical Bond Lengths and Dihedral Angles between the Innermost Rings for 6T and 6F

\begin{tabular}{|c|c|c|c|c|c|c|}
\hline method & outer ring double & ring single & inner ring double & inter ring & BLA & dihedral \\
\hline \multicolumn{7}{|c|}{$6 \mathrm{~T}$} \\
\hline $\operatorname{exptl}^{66}$ & $1.370 / 1.376$ & $1.400 / 1.403$ & $1.367 / 1.380$ & 1.444 & 0.070 & 180.0 \\
\hline LSDA/6-311G* & 1.378 & 1.397 & 1.378 & 1.421 & 0.043 & 163.4 \\
\hline LC-SVWN/6-311G* & 1.352 & 1.411 & 1.352 & 1.451 & 0.099 & 149.0 \\
\hline LC-SVWN5/6-311G* & 1.353 & 1.413 & 1.353 & 1.453 & 0.100 & 148.6 \\
\hline $\mathrm{BLYP} / 6-311 \mathrm{G}^{*}$ & 1.392 & 1.416 & 1.393 & 1.443 & 0.050 & 163.1 \\
\hline LC-BLYP/6-311G* & 1.353 & 1.413 & 1.353 & 1.450 & 0.097 & 150.6 \\
\hline B3LYP/6-311G* & 1.378 & 1.413 & 1.378 & 1.443 & 0.065 & 158.5 \\
\hline CAM-B3LYP/6311G* & 1.366 & 1.416 & 1.366 & 1.450 & 0.084 & 152.2 \\
\hline BNL-0.182/6-311G* & 1.400 & 1.440 & 1.400 & 1.473 & 0.073 & 149.9 \\
\hline LC-BP86/6311G* & 1.354 & 1.409 & 1.354 & 1.446 & 0.092 & 151.2 \\
\hline B3P86/6-311G* & 1.376 & 1.408 & 1.376 & 1.438 & 0.062 & 159.2 \\
\hline B3P $86-30 / 6-311 G^{*}$ & 1.371 & 1.409 & 1.371 & 1.441 & 0.070 & 156.9 \\
\hline PBE1PBE/6-311G* & 1.375 & 1.409 & 1.375 & 1.440 & 0.065 & 158.3 \\
\hline LC-PBEPBE/6-311G* & 1.354 & 1.408 & 1.354 & 1.446 & 0.092 & 150.7 \\
\hline LC-wPBE/6-311G* & 1.362 & 1.416 & 1.362 & 1.456 & 0.094 & 149.1 \\
\hline M06/6-311G* & 1.371 & 1.408 & 1.371 & 1.439 & 0.068 & 158.4 \\
\hline M06-HF/6-311G* & 1.364 & 1.426 & 1.365 & 1.462 & 0.098 & 146.6 \\
\hline wB97/6-311G* & 1.368 & 1.424 & 1.367 & 1.463 & 0.095 & 150.9 \\
\hline wB97x/6-311G* & 1.366 & 1.420 & 1.366 & 1.458 & 0.091 & 150.7 \\
\hline wB97xd/6-311G* & 1.368 & 1.417 & 1.368 & 1.453 & 0.084 & 150.2 \\
\hline wB97xd/6-31G* & 1.371 & 1.418 & 1.371 & 1.452 & 0.081 & 154.4 \\
\hline OLYP/6-311G* & 1.393 & 1.410 & 1.390 & 1.442 & 0.053 & 160.8 \\
\hline LC-OLYP/6-311G* & 1.352 & 1.410 & 1.352 & 1.448 & 0.096 & 150.2 \\
\hline TPSSTPSS/6-311G* & 1.389 & 1.411 & 1.389 & 1.438 & 0.049 & 169.3 \\
\hline LC-TPSSTPSS/6-311G* & 1.353 & 1.409 & 1.353 & 1.447 & 0.094 & 150.2 \\
\hline $\mathrm{HF} / 6-311 \mathrm{G}^{*}$ & 1.351 & 1.429 & 1.351 & 1.462 & 0.112 & 148.3 \\
\hline $\mathrm{MP} 2 / 6-311 \mathrm{G}^{*}$ & 1.393 & 1.409 & 1.393 & 1.447 & 0.054 & 145.7 \\
\hline $\mathrm{MP} 2 / 6-311 \mathrm{G}^{*}$ & 1.391 & 1.405 & 1.391 & 1.439 & 0.049 & 180.0 \\
\hline \multicolumn{7}{|c|}{$6 \mathrm{~F}$} \\
\hline exptl $1^{45}$ & 1.362 & 1.408 & 1.358 & 1.432 & 0.074 & 180 \\
\hline LSDA/6-311G* & 1.372 & 1.405 & 1.372 & 1.411 & 0.039 & 180 \\
\hline $\mathrm{BLYP} / 6-311 \mathrm{G}^{*}$ & 1.386 & 1.424 & 1.387 & 1.431 & 0.044 & 180 \\
\hline LC-BLYP/6-311G* & 1.347 & 1.420 & 1.347 & 1.436 & 0.089 & 180 \\
\hline B3LYP/6-311G* & 1.372 & 1.420 & 1.372 & 1.429 & 0.057 & 180 \\
\hline CAM-B3LYP/6311G* & 1.361 & 1.422 & 1.361 & 1.432 & 0.071 & 180 \\
\hline B3P86/6-311G* & 1.370 & 1.415 & 1.370 & 1.425 & 0.055 & 180 \\
\hline B3P86-30/6-311G* & 1.365 & 1.416 & 1.365 & 1.427 & 0.062 & 180 \\
\hline wB97xd/6-311G* & 1.362 & 1.423 & 1.362 & 1.437 & 0.075 & 180 \\
\hline
\end{tabular}

Bond Lengths of Thiophene and Furan Oligomers. The double bond lengths in the inner rings of $6 \mathrm{~T}$ single crystals vary from 1.367 to $1.380 \AA \AA^{66}$ The ring single bond lengths of the two inner rings are $1.400 \AA$ and $1.403 \AA$, and the central inter-ring single bond length is $1.444 \AA$. The BLA is therefore $\sim 0.07 \AA$.
Bond lengths in $8 \mathrm{~T}^{67}$ are as follows: double bonds of inner rings,

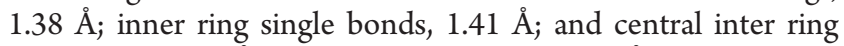
single bond, $1.43 \AA$. BLA in $8 \mathrm{~T}$ is therefore $0.05 \AA$. Because there is little difference between gas-phase structures of $6 \mathrm{~T}$ and $8 \mathrm{~T}$ at the B3LYP/6-311G* level, part of the differences between 


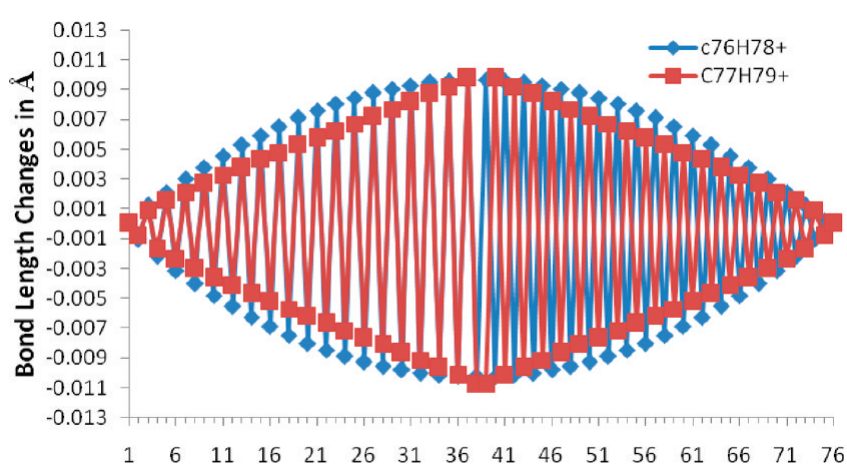

Figure 3. Differences in $\mathrm{C}-\mathrm{C}$ bond lengths between $\mathrm{C}_{76} \mathrm{H}_{78}$ and $\mathrm{C}_{76} \mathrm{H}_{78}{ }^{+}$and between $\mathrm{C}_{76} \mathrm{H}_{78}$ and $\mathrm{C}_{77} \mathrm{H}_{79}{ }^{+}$at $\mathrm{BLYP} / 6-31 \mathrm{G}^{*}$.

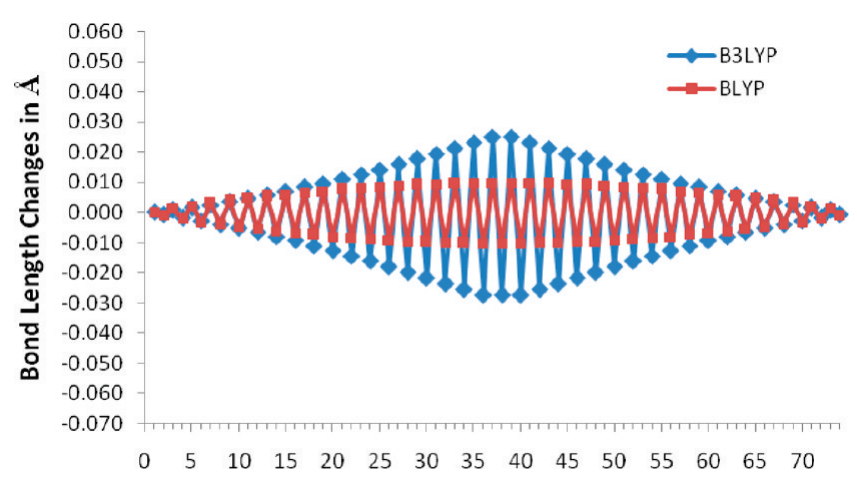

Figure 4. Differences in $\mathrm{C}-\mathrm{C}$ bond lengths between $\mathrm{C}_{76} \mathrm{H}_{78}$ and $\mathrm{C}_{75} \mathrm{H}_{77}{ }^{+}$at BLYP/6-31G* and B3LYP/6-31G*.

experimental data of $6 \mathrm{~T}$ and $8 \mathrm{~T}$ might be due to experimental uncertainties. $6 \mathrm{~F}$ has slightly shorter inner-ring double bonds (1.358/1.362 $\AA$ ) and a shorter inter-ring single bond (1.432 $\AA$ ) than $6 \mathrm{~T}$. The central inter-ring single bond in $6 \mathrm{~F}$ is $1.408-\AA$ long. ${ }^{45} \mathrm{BLA}$ is thus $0.074 \AA$, slightly larger than in 6T. In Table 1 , bond lengths of fully optimized nonplanar structures of $6 \mathrm{~T}$ and of planar 6F at various levels of theory are summarized. Planarization has a little effect on the bond lengths in 6T.

The general trends are as follows: pure DFT (LSDA) tends to overestimate double bond lengths and to underestimate single bond lengths. BLA is therefore too small. Gradient correction (BLYP) predicts single and double bonds to be too long. Hybrid functionals (especially B3P86-30\% and M06) are very accurate. Long-range correction shortens double bonds and comes sometimes close to values obtained with HF theory. Single bond lengths are overestimated but not as much as with HF theory. As a result, BLA is too large with all range-separated functionals. CAM-B3LYP with only $65 \%$ of long-range HF exchange and $\gamma=$ 0.33 and $w B 97 x d$ with a rather large short-range part $(\gamma=0.2)$ are doing better than the LC-functionals with $100 \%$ HF exchange and $\gamma=0.4$. CAM-B3LYP and wB97XD perform better for $6 \mathrm{~F}$ than for $6 \mathrm{~T}$. BNL predicts all bonds to be too long, but BLA is accurate. HF theory overestimates BLA because it overestimates single bond lengths and underestimates double bond lengths. Correlation at the MP2 levels shortens the double bonds correctly but lengthens the single bonds too much. The closest agreement between theory and experiment for $6 \mathrm{~T}$ is reached with global hybrid functionals. For 6F, B3P86-30\%, CAM-B3LYP, and $\mathrm{wB} 97 \mathrm{XD}$ give the best results.

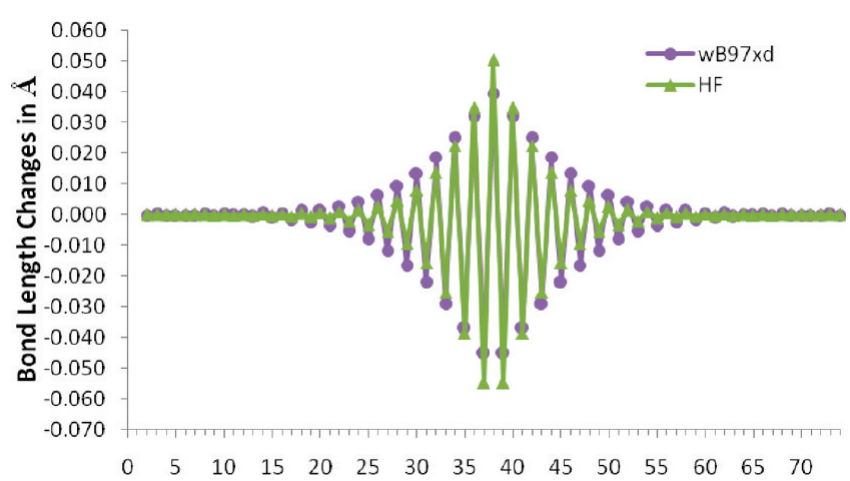

Figure 5. Differences in $\mathrm{C}-\mathrm{C}$ bond lengths between $\mathrm{C}_{76} \mathrm{H}_{78}$ and $\mathrm{C}_{75} \mathrm{H}_{77}{ }^{+}$at wB97XD/6-31G* and $\mathrm{HF} / 6-31 \mathrm{G}^{*}$.

Defect Sizes. Defect sizes of $\mathrm{C}_{41} \mathrm{H}_{43}{ }^{+}, \mathrm{C}_{101} \mathrm{H}_{103}{ }^{+}$, and $19 \mathrm{~T}^{+}$ were previously investigated with pure DFT and with the B3P86-30\% global hybrid functional using StevensBach-Krauss pseudopotentials (SBK). ${ }^{12,13,24}$ Here, we are repeating some of the calculations with range-separated hybrids, i.e., LC-wPBE and $w B 97 X D$, and with the "BLYP series", i.e., BLYP, B3LYP, CAM-B3LYP, and LC-BLYP. Because of the size of the systems, 6-31G* and SBK basis sets were used. Defect sizes decrease by about six bonds (or about one ring) with $6-31 G^{*}$ compared to $\mathrm{SBK}$ in $19 \mathrm{~T}^{+}$and $\mathrm{C}_{77} \mathrm{H}_{79}{ }^{+}$with the wB97XD functional. Differences in bond lengths between 6-311G* and 6-31G* basis sets can be seen in Table 1 for wB97XD. 6-31G* lengthens the double bonds and slightly shortens the single bonds compared to $6-311 \mathrm{G}^{*}$.

Test calculations were done on $19 \mathrm{~T}^{+}$and $25 \mathrm{~T}^{+}$and on $\mathrm{C}_{75} \mathrm{H}_{77}{ }^{+}$and $\mathrm{C}_{101} \mathrm{H}_{103}{ }^{+}$to determine the required chain lengths for converged defect size. With LC functionals, defect sizes are converged for $19 \mathrm{~T}^{+}$and $\mathrm{C}_{75} \mathrm{H}_{77}{ }^{+}$. With global hybrids, defects are delocalized and do not converge with increasing chain length. Figure 3 shows differences in $\mathrm{C}-\mathrm{C}$ bond lengths between neutral $\mathrm{C}_{76} \mathrm{H}_{78}$ and $\mathrm{C}_{76} \mathrm{H}_{78}{ }^{+}$and $\mathrm{C}_{77} \mathrm{H}_{79}{ }^{+}$cations with BLYP. The expectation value of the spin operator $\left(\left\langle S^{2}\right\rangle\right)$ for $\mathrm{C}_{76} \mathrm{H}_{78}{ }^{+}$is 0.77. Figure 3 shows that odd- and even-numbered systems have similar defect extensions and shapes when the wave function of the open-shell system is not spin-contaminated. As soon as HF exchange is included, unreasonably high $\left\langle S^{2}\right\rangle$ values are obtained for long polyene cations, while global hybrids produce good $\left\langle S^{2}\right\rangle$ values for thiophene oligomer cations. ${ }^{12,13}$ Spin contamination is avoided with polyenes by employing odd-numbered systems and investigating charged defects with closed-shell calculations.

The effect of HF exchange on defect size is established in Figures 4 (BLYP and B3LYP) and 5 (wB97XD and HF) for $\mathrm{C}_{75} \mathrm{H}_{77}{ }^{+}$(bond lengths are compared to those of $\mathrm{C}_{76} \mathrm{H}_{78}$, from which the innermost bond was deleted to achieve the same chain length and position of double bonds as in $\mathrm{C}_{75} \mathrm{H}_{77}{ }^{+}$). Increasing HF exchange leads to defect localization. While defects are delocalized at BLYP and B3LYP, they spread over about 48 bonds (SBK) and 42 bonds $\left(6-31 G^{*}\right)$ with the wB97XD functional and over about 30 bonds at the HF/6-31G* level. The criterion for determining defect size is that bond lengths in the cation differ by more than $0.002 \AA$ from those of the neutral molecule.

Oligothiophene cations have one unpaired electron and have to be treated in the open-shell formalism. The $\left\langle S^{2}\right\rangle$ values for $19 \mathrm{~T}^{+}$are BLYP, 0.75; B3LYP, 0.76; CAM-B3LYP, 0.88; wB97XD, 0.90; LC-wPBE, 1.09; and LC-BLYP, 1.11. The values 


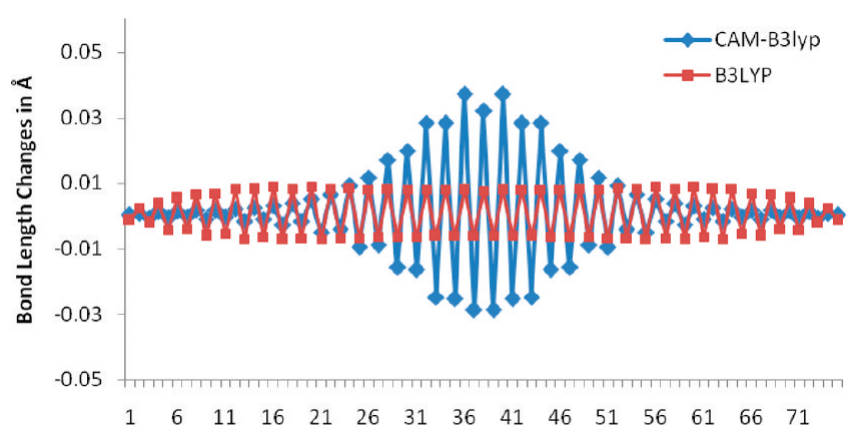

Figure 6. Differences in bond length between $19 \mathrm{~T}$ and $19 \mathrm{~T}^{+}$at $\mathrm{B} 3 \mathrm{LYP} /$ SBK (red squares) and CAM-B3LYP/SBK (blue diamonds).

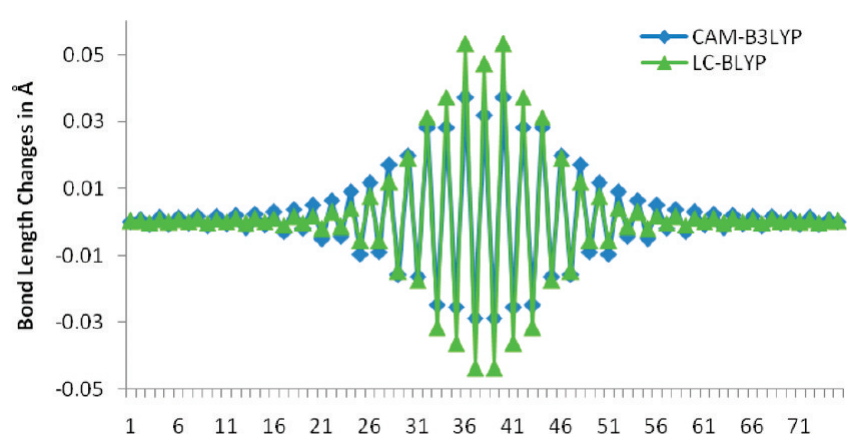

Figure 7. Differences in bond length between $19 \mathrm{~T}$ and $19 \mathrm{~T}^{+}$at CAMB3LYP/SBK (blue diamonds) and LC-BLYP/SBK (green triangles).

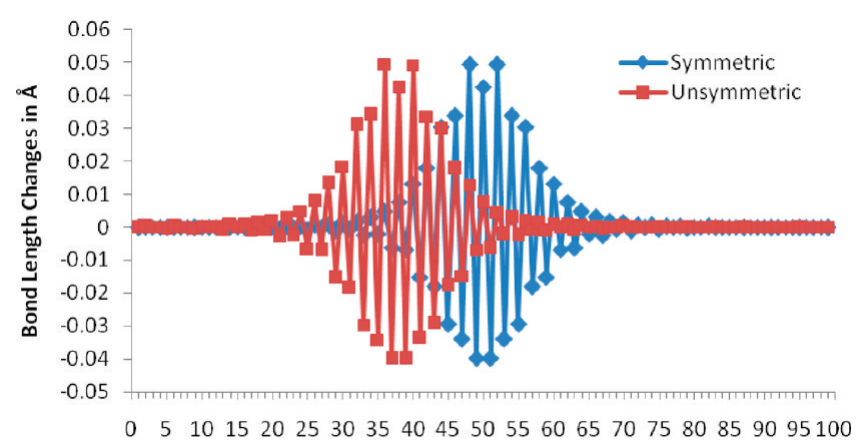

Figure 8. Localized defects at LC-wPBE/SBK in different positions on $25 \mathrm{~T}^{+}$.

do not increase from $19 \mathrm{~T}^{+}$to $25 \mathrm{~T}^{+}$. As shown in Figure 2, cations are nonplanar with range-separated hybrid functionals. The planarization energy is very small, however, e.g., $2.24 \mathrm{kcal} / \mathrm{mol}$ for $19 \mathrm{~T}^{+}$at wB97XD/6-31 $\mathrm{G}^{*}$. Because the defect sizes are virtually the same in planar and nonplanar forms at wB97XD/6-31G*, planar cations were used in the following. In Figure 6, bond length changes upon ionization in $19 \mathrm{~T}^{+}$are shown at the B3LYP/SBK and CAM-B3LYP/SBK levels and in Figure 7 at CAM-B3LYP/ SBK and LC-BLYP/SBK. The inclusion of large amounts of longrange HF exchange changes the defect shape and extension from totally delocalized at B3LYP with a tendency to split into two separate defects to a localized defect with a maximum in the middle of the chain. LC correction to BLYP localizes the defect more than CAM-B3LYP. LC-wPBE results (not shown) are almost identical to those with LC-BLYP. With the LC correction and with wB97XD, defects spread over about 9 or 10 thiophene rings. With

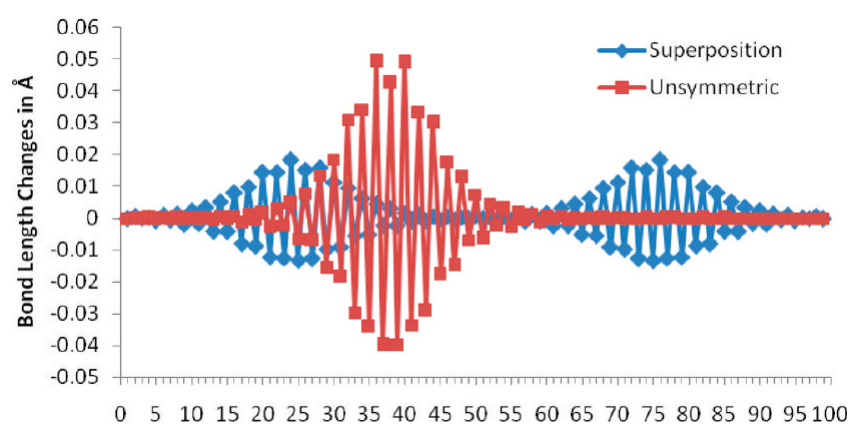

Figure 9. Two defect types on $25 \mathrm{~T}^{+}$at the $\mathrm{LC}$-wPBE/SBK level.

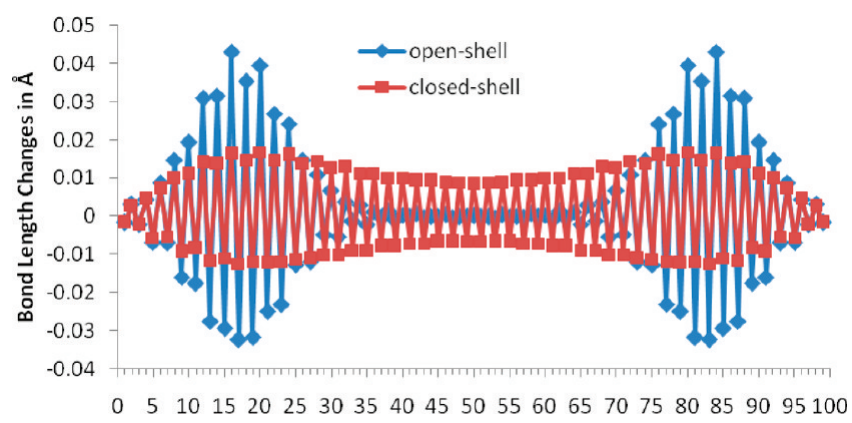

Figure 10. Comparison of defects on $25 \mathrm{~T}^{2+}$ with closed- and open-shell calculations at the wB97XD/SBK level of theory.

CAM-B3LYP, the defects spread over about 15 rings. The defect size in $19 \mathrm{~F}^{+}$is 10 rings at $\mathrm{wB} 97 \mathrm{XD} / 6-31 \mathrm{G}^{*}$.

As demonstrated in Figure 8, it is possible to move the defect from the middle of the chain closer to one end. This move changes neither the energy of the cation nor the defect size and shape. Therefore, defects are converged with respect to chain length with the LC-corrected functionals.

It is also possible to obtain cations that exhibit two separated defects, as seen in Figure 9 at LC-wPBE/SBK. A cation with two half charges at either end of the chain is reminiscent of the DFT problem with the unphysical dissociation of symmetrical radical cations into two fragments with half charges. Such a state might be a superposition of two nonsymmetrical cations and a valid solution of the Schrödinger equation, as discussed by Vydrov and Scuseria ${ }^{49}$ for symmetrical radical cations. The problem with pure DFT is that it underestimates the energy of such solutions and predicts them to be global minima, whereas they should be energetically degenerate with the radical/cation pair. HF, in contrast, overestimates the energy of the linear combination. At LC-wPBE, the split charge state lies $12 \mathrm{kcal} / \mathrm{mol}$ above the single defect cation, suggesting that the problem is overcorrected (too close to the HF result).

Defects in Dications. Geometries of $25 \mathrm{~T}$ dications were optimized with LC-wPBE and wB97XD functionals. Figure 10 shows the defects obtained with closed-shell and open-shell $\left(\left\langle S^{2}\right\rangle=1.44\right)$ calculations at wB97XD/SBK. It is clearly visible that even with closed-shell calculations there is a tendency for splitting the defect and placing the two charges at opposite ends of the molecule. Open-shell calculations lead to a complete separation of the two charges with an undistorted chain segment in the middle. Figure 11 shows that LC-wPBE $\left(\left\langle S^{2}\right\rangle=1.98\right)$ leads to defects virtually identical to those of $w B 97 \mathrm{XD} / \mathrm{SBK}$. With both methods, the defect size is nine rings. 


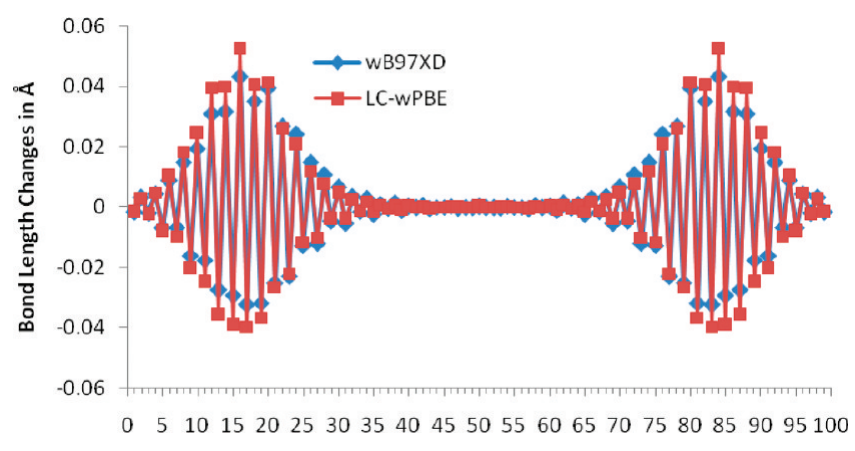

Figure 11. Comparison of defects on $25 \mathrm{~T}^{2+}$ with open-shell calculations at the $\mathrm{wB} 97 \mathrm{XD} / \mathrm{SBK}$ and LC-wPBE/SBK levels of theory.

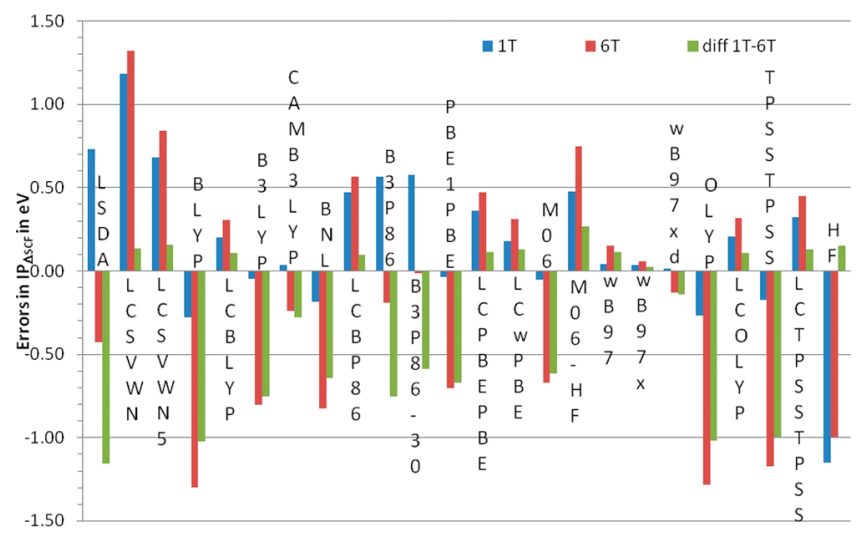

Figure 12. Errors in $\mathrm{IP}_{\triangle \mathrm{SCF}}$ of $1 \mathrm{~T}$ and $6 \mathrm{~T}$ at various levels of theory.

Ionization Energies. Gas phase ionization energies of neutral $1 \mathrm{~T}$ through $5 \mathrm{~T}$ were measured by Jones et al., ${ }^{68}$ for $3 \mathrm{~T}$ through $8 \mathrm{~T}$ by da Silva Filho et al., ${ }^{69}$ and for 1F through 4F by Distefano et al. ${ }^{70}$ It was established recently ${ }^{14}$ that $\mathrm{IP}_{\triangle \mathrm{SCF}}$ 's of $1 \mathrm{~T}, 1 \mathrm{~F}$, and pyrrole are predicted quantitatively at the B3LYP/6-311G* level and that the higher IPs can be obtained by adding the TDB3LYP excitation energies of the cation to $\mathrm{IP}_{\triangle \mathrm{SCF}}$. For longer oligomers, the accuracy declines because B3LYP, like any other global hybrid functional, increasingly underestimates $\mathrm{IP}_{\triangle \mathrm{SCF}}$ with growing chain length. To find out whether range-separated hybrid functionals improve the chain length dependence, vertical $\mathrm{IP}_{\triangle \mathrm{SCF}}$ 's were calculated for $1 \mathrm{~T}$ and $6 \mathrm{~T}$. In Figure 12, the errors compared to the experimental IPs of $8.87 \mathrm{eV}(1 \mathrm{~T})^{68}$ and $6.98 \mathrm{eV}$ $(6 \mathrm{~T})^{69}$ are plotted. $1 \mathrm{~T}$ entries include a zero point energy (ZPE) correction of $0.059 \mathrm{eV}$. For $6 \mathrm{~T}$, the ZPE correction is negligibly small. The third (green) bar shows the difference in errors between the $\mathrm{IP}_{\triangle \mathrm{SCF}}$ 's of $1 \mathrm{~T}$ and $6 \mathrm{~T}$. A short green bar indicates therefore correct chain length dependence. Figure 12 reveals that all range-separated functionals with the exception of BNL have similar errors for $1 \mathrm{~T}$ and $6 \mathrm{~T}$ and therefore the correct chain length dependence. The absolute $\mathrm{IP}_{\Delta \mathrm{SCF}}$ 's, however, are not necessarily correct. The functionals that predict $\mathrm{IP}_{\triangle \mathrm{SCF}}$ 's of $1 \mathrm{~T}$ and $6 \mathrm{~T}$ within about $0.3 \mathrm{eV}$ of the experiment are LC-BLYP, CAM-B3LYP (with some problems with chain lengths dependence), LC-wPBE, the wB97 series, and LC-OLYP.

Figure 13 depicts the chain length dependence of B3LYP, BNL, CAM-B3LYP, wB97xd, and LC-BLYP IP $\triangle$ SCF $_{\text {'s compared }}$ to experimental results. B3LYP IP ${ }_{\triangle S C F}$ 's fall off too fast, and BNL follows this trend. The reason is probably that with BNL, $\gamma$ tuning by matching $\mathrm{IP}_{\triangle \mathrm{SCF}}$ and $-\varepsilon_{\mathrm{HOMO}}$ energy requires smaller

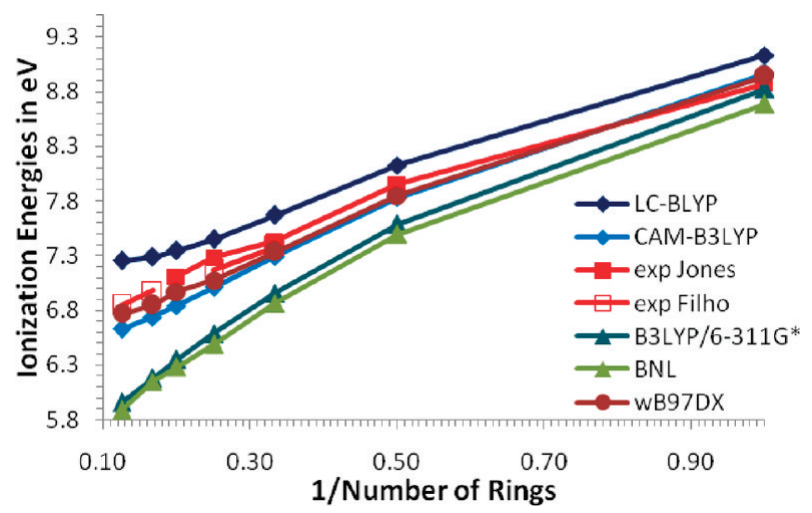

Figure 13. Chain length dependence of $\mathrm{IP}_{\triangle \mathrm{SCF}}$ of thiophene oligomers.

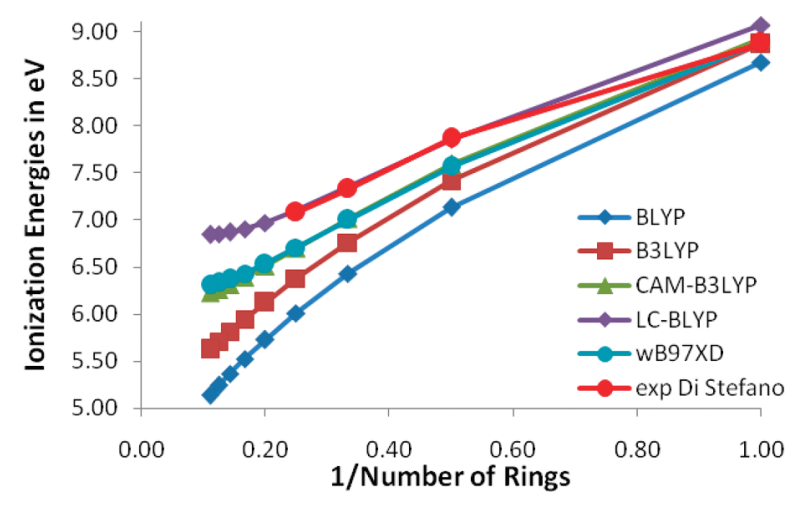

Figure 14. Chain length dependence of $\mathrm{IP}_{\triangle \mathrm{SCF}}$ of furan oligomers.

values with increasing chain length (for 1T, $\gamma=0.352$; and for $8 \mathrm{~T}$, $\gamma=0.168)$. Thus, the short-range part increases for longer oligomers, and results get closer to those with global hybrids. CAM-B3LYP and wB97xd with constant $\gamma$ are very close to experimental results and have the correct chain length dependence. LC-BLYP predicts values slightly above experimental values and a leveling off at a relatively short chain length. Such a convergence is not seen in the experimental data. $\mathrm{IP}_{\triangle \mathrm{SCF}}$ 's extrapolated to infinite chain length with second degree polynomial fits are as follows exptl, $6.45 \mathrm{eV}$; CAM-B3LYP, $6.22 \mathrm{eV}$; wB97xd, $6.48 \mathrm{eV}$; LC-BLYP, $7.08 \mathrm{eV}$.

For furan oligomers IPs are available only up to a chain length of 4 rings. ${ }^{70}$ Figure 14 compares the $\mathrm{IP}_{\triangle \mathrm{SCF}}$ of $1-9 \mathrm{~F}$ with several functionals to these experimental values. The first IP of the monomer is predicted quantitatively with B3LYP, CAM-B3LYP, and $\mathrm{wB} 97 \mathrm{XD}$. However, in contrast to results for thiophene oligomers, CAM-B3LYP and ${ }^{\mathrm{w} B}$ 97XD slightly overestimate the decrease with increasing chain length and underestimate the $\mathrm{IP}_{\triangle \mathrm{SCF}}$ of long oligomers. LC-BLYP slightly overestimates the IP of the monomer but agrees quantitatively with experimental results for the longer oligomers. LC-BLYP again predicts the onset of convergence, while B3LYP and BLYP predicta accelerated decrease of $\mathrm{IP}_{\triangle \mathrm{SCF}}$ 's with increasing chain length. Experimental IPs ${ }^{70}$ extrapolate to about $6.4 \mathrm{eV}, \mathrm{LC}-\mathrm{BLYP}$ to $6.66 \mathrm{eV}$, wB97XD to $5.98 \mathrm{eV}$, and CAM-B3LYP to $5.82 \mathrm{eV}$.

Excitation Energies and Optical Band Gaps. UV spectra become simpler as oligomers get larger. Because we are interested in long oligomers and polymers, monomers are not representative and were not considered. In Figure 15, errors in 
the first excitation energies of $2 \mathrm{~T}$ and $6 \mathrm{~T}$ are plotted. The experimental $E_{\mathrm{g}}$ 's are $4.09 \mathrm{eV}^{71-73}$ for $2 \mathrm{~T}$ and $2.84^{71,72}$ or $2.92^{74} \mathrm{eV}$ for $6 \mathrm{~T}$. Solvent effects were shown theoretically to be small in ref 13. To calculate the errors, we used $4.19 \mathrm{eV}$ as suggested by Andrzejak and Witek ${ }^{73}$ as the vertical excitation energy for $2 \mathrm{~T}$ and $2.92 \mathrm{eV}$ for $6 \mathrm{~T}$.

For $2 \mathrm{~T}$, the HOMO-LUMO $\left(1-1^{\prime}\right)$ transition is the leading electron configuration of the first excited state with a CI

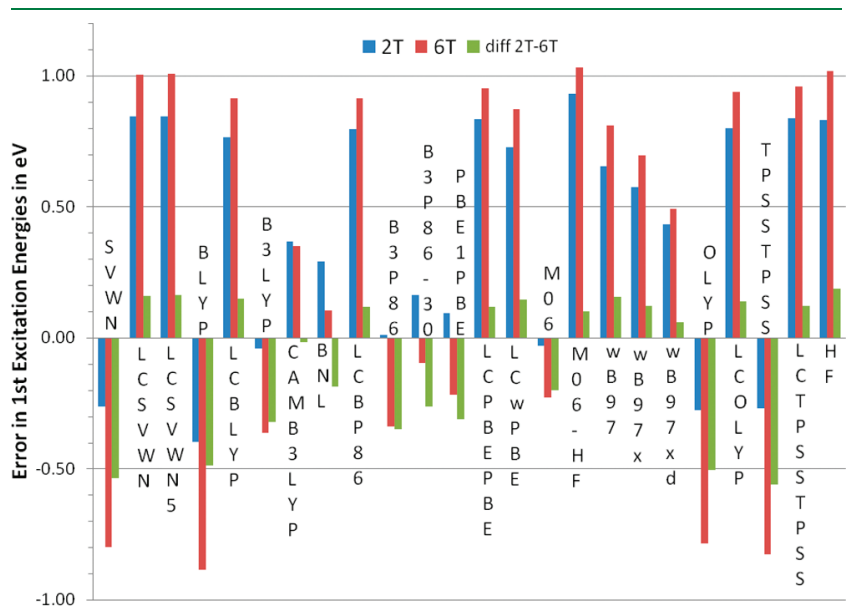

Figure 15. Errors in first excitation energies of neutral $2 \mathrm{~T}$ and $6 \mathrm{~T}$ at various levels of theory. coefficient between 0.68 and 0.70 with all density functionals and at the HF level. There is no other contribution with a CI coefficient above 0.1 . Double excitations, which are not included in TDDFT, are of little importance in these systems according to CASSCF calculations. ${ }^{75-77}$ The oscillator strength of the first excitation energy is between 0.38 and 0.40 at all levels of theory employed.

For the first excited state of 6T, pure DFT and global hybrids predict the $1-1^{\prime}$ transition to be the only electronic configuration with a CI coefficient above 0.1 ; CI coefficients range from 0.69 to 0.71 . At the HF level, the $1-1^{\prime}$ transition has a CI coefficient of 0.58 , and there are contributions arising from $\mathrm{HOMO}-1$ to LUMO+1 $\left(2-2^{\prime}\right)$ and HOMO -2 to $\mathrm{LUMO}+2$ $\left(3-3^{\prime}\right)$ with CI coefficients of 0.27 and 0.16 . Range-separated functionals produce values very close to those of HF theory. Estimates of the oscillator strength range from 1.58 to 1.94 (Table 2).

Pure DFT underestimates excitation energies, and errors get larger with increasing chain length. Hybrid functionals are quite accurate for short oligomers, but the chain length dependence is wrong. Long-range correction leads to proper chain length dependence, but excitation energies are too high. Three functionals are better than the rest: B3P $86-30 \%$ with the smallest absolute errors for $2 \mathrm{~T}$ and $6 \mathrm{~T}$ of +0.16 and $-0.10 \mathrm{eV}$ but the wrong chain length dependence because of the opposite signs of the errors, CAM-B3LYP with errors of 0.37 and $0.35 \mathrm{eV}$, and wB97xd with errors 0.43 and $0.49 \mathrm{eV}$.

Table 2. Spectroscopic Data (Energy $(E)$ and Oscillator Strength $(f)$, for $6 \mathrm{~T}$ and $6 \mathrm{~T}^{+}$, and $\left\langle S^{2}\right\rangle$ for $6 \mathrm{~T}^{+}$; energies in $\mathrm{eV}$, basis set: 6-311G*)

\begin{tabular}{|c|c|c|c|c|c|c|c|c|c|}
\hline \multirow{3}{*}{ exptl } & \multicolumn{2}{|c|}{$6 \mathrm{~T}$} & \multirow[t]{2}{*}{$6 \mathrm{~T}^{+}$} & \multicolumn{3}{|c|}{$6 \mathrm{~T}^{+} 1$ st excited state } & \multicolumn{3}{|c|}{$6 \mathrm{~T}^{+}$2nd excited state } \\
\hline & \multicolumn{2}{|c|}{$2.85,^{72} 2.92^{74}$} & & \multicolumn{3}{|c|}{$0.84^{74,79}$} & \multicolumn{3}{|c|}{$1.59^{74,79}$} \\
\hline & $E$ & $f$ & $\left\langle S^{2}\right\rangle$ & E & $f$ & $\left\langle S^{2}\right\rangle$ & $E$ & $f$ & $\left\langle S^{2}\right\rangle$ \\
\hline LSDA & 2.121 & 1.58 & 0.75 & 0.914 & 0.34 & 0.75 & 1.746 & 1.35 & 0.76 \\
\hline LC-SVWN & 3.924 & 1.90 & 0.95 & 1.222 & 1.17 & 0.95 & 2.120 & 1.11 & 1.31 \\
\hline LC-SVWN5 & 3.929 & 1.90 & 0.96 & 1.231 & 1.19 & 0.97 & 2.082 & 1.07 & 1.34 \\
\hline BLYP & 2.036 & 1.62 & 0.75 & 0.864 & 0.25 & 0.75 & 1.646 & 1.75 & 0.76 \\
\hline LC-BLYP & 3.835 & 1.88 & 0.98 & 1.231 & 1.23 & 0.99 & 2.018 & 1.01 & 1.38 \\
\hline B3LYP & 2.557 & 1.86 & 0.77 & 0.942 & 0.45 & 0.76 & 1.786 & 1.78 & 0.88 \\
\hline CAM-B3LYP & 3.271 & 1.94 & 0.85 & 1.042 & 0.82 & 0.82 & 1.904 & 1.49 & 1.13 \\
\hline BNL-0.182 & 3.025 & 1.86 & 0.78 & 0.908 & 0.94 & & 2.001 & 1.47 & \\
\hline LC-BP86 & 3.835 & 1.92 & 0.98 & 1.218 & 1.25 & 0.99 & 2.063 & 1.00 & 1.42 \\
\hline BP86 & 2.079 & 1.61 & 0.75 & 0.880 & 0.29 & 0.75 & 1.678 & 1.68 & 0.76 \\
\hline B3P86 & 2.833 & 1.88 & 0.77 & 0.953 & 0.48 & 0.76 & 1.828 & 1.74 & 0.89 \\
\hline В3P86-30 & 2.822 & 1.95 & 0.80 & 0.987 & 0.57 & 0.77 & 1.824 & 1.73 & 1.01 \\
\hline PBE1PBE & 2.704 & 1.92 & 0.78 & 0.969 & 0.54 & 0.77 & 1.835 & 1.72 & 0.96 \\
\hline LC-PBEPBE & 3.871 & 1.94 & 0.98 & 1.220 & 1.27 & 0.99 & 2.072 & 0.98 & 1.44 \\
\hline LC-wPBE & 3.794 & 1.92 & 0.98 & 1.188 & 1.21 & 0.98 & 2.005 & 1.01 & 1.43 \\
\hline M06 & 2.691 & 1.85 & 0.78 & 0.934 & 0.52 & 0.77 & 1.801 & 1.67 & 0.94 \\
\hline wB97 & 3.729 & 1.91 & 0.94 & 1.159 & 1.13 & 0.94 & 2.063 & 1.11 & 1.33 \\
\hline wB97x & 3.617 & 1.93 & 0.91 & 1.116 & 1.03 & 0.89 & 2.036 & 1.24 & 1.24 \\
\hline wB97xd & 3.411 & 1.96 & 0.86 & 1.048 & 0.87 & 0.83 & 1.998 & 1.45 & 1.12 \\
\hline OLYP & 2.136 & 1.62 & 0.75 & 0.893 & 0.31 & 0.75 & 1.707 & 1.71 & 0.76 \\
\hline LC-OLYP & 3.858 & 1.90 & 0.98 & 1.231 & 1.24 & 0.99 & 2.042 & 1.01 & 1.39 \\
\hline TPSSTPSS & 2.093 & 1.71 & 0.75 & 0.898 & 0.29 & 0.76 & 1.703 & 1.80 & 0.78 \\
\hline LC-TPSSTPSS & 3.880 & 1.93 & 1.01 & 1.237 & 1.33 & 1.02 & 2.018 & 0.92 & 1.48 \\
\hline $\mathrm{HF}$ & 3.938 & 1.87 & 3.03 & 1.033 & 1.86 & 3.85 & 5.440 & 0.13 & 3.54 \\
\hline
\end{tabular}




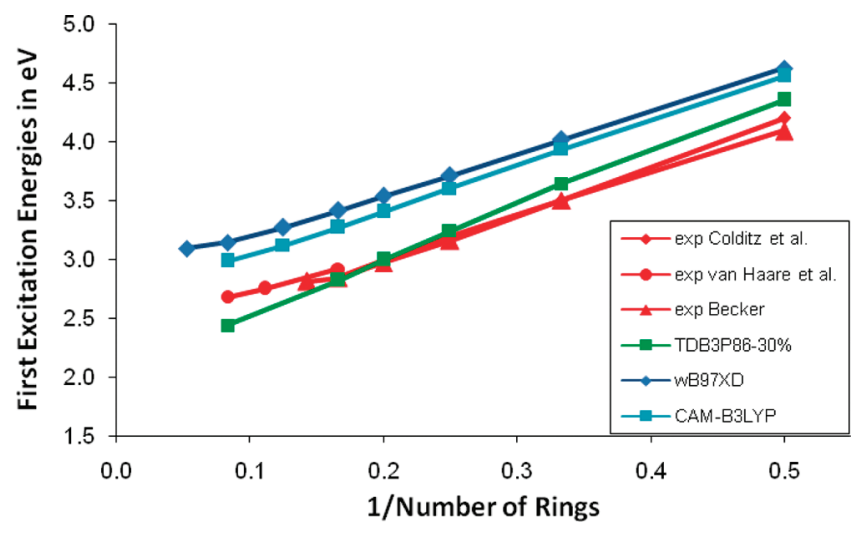

Figure 16. First excitation energies of neutral thiophene oligomers with different density functionals compared to experimental results. ${ }^{71,72,74}$

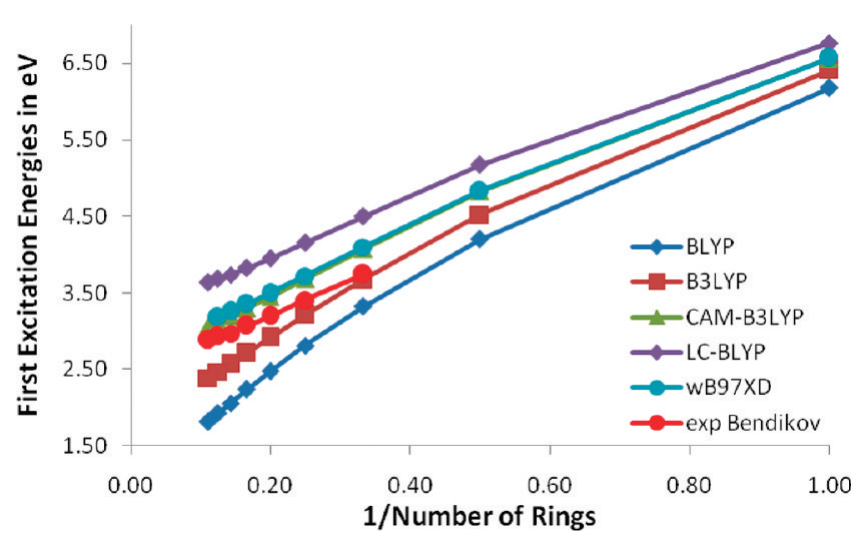

Figure 17. First excitation energies of neutral furan oligomers with different density functionals compared to experimental results. ${ }^{45}$

In Figure 16, excitation energies of $2 \mathrm{~T}-12 \mathrm{~T}$ with B3P86-30\%, CAM-B3LYP, and wB97XD are plotted versus inverse chain length. The extrapolated excitation energies (linear and polynomial fits give almost the same results) are $2.43 \mathrm{eV}$ with the solution data of Colditz et al., ${ }^{72} 2.47 \mathrm{eV}$ according to the solution data of van Haare et al., ${ }^{74} 2.85 \mathrm{eV}$ at wB97XD, 2.67 with CAM-B3LYP, and $2.00 \mathrm{eV}$ at B3P86-30\%.

The electronic configurations of excited states of furan oligomers are very similar to those described above for $2 \mathrm{~T}$ and $6 \mathrm{~T}$. The excitation energies are less underestimated with global hybrids, and the errors with CAM-B3LYP and wB97XD are smaller than for thiophene oligomers (Figure 17). Extrapolation with second degree polynomial fits predicts the following $\varepsilon_{\max }$ values for polyfuran: exptl, ${ }^{45} 2.53 \mathrm{eV}$; CAM-B3LYP, $2.55 \mathrm{eV}$; and wB97XD, $2.65 \mathrm{eV}$. LC-BLYP overestimates first excitation energies of neutral oligomers by $0.75 \mathrm{eV}$ and extrapolates to $3.22 \mathrm{eV}$. BLYP and B3LYP predict polymer values of 0.92 and $1.67 \mathrm{eV}$.

Excitation Energies of Cations. The $2 \mathrm{~T}$ cation was produced in a matrix at $77 \mathrm{~K}$. It has a strong and a very weak absorption in the UV spectrum. ${ }^{78} 2 \mathrm{~T}^{+}$and $3 \mathrm{~T}^{+}$cannot be produced in solution. $3 \mathrm{~T}$ seems to dimerize upon oxidation as the recorded absorption spectrum is identical to that of $6 \mathrm{~T}^{+} .{ }^{70}$ Starting with $4 \mathrm{~T}$, cations were observed in solution. ${ }^{74,79}$ Upon oxidation of $4 \mathrm{~T}$ to $9 \mathrm{~T},{ }^{74,79}$ the absorption of the neutral form disappears, and the two new features appear at lower energy. Both bands decrease in energy with increasing chain length. For $12 \mathrm{~T}$, the absorption of the

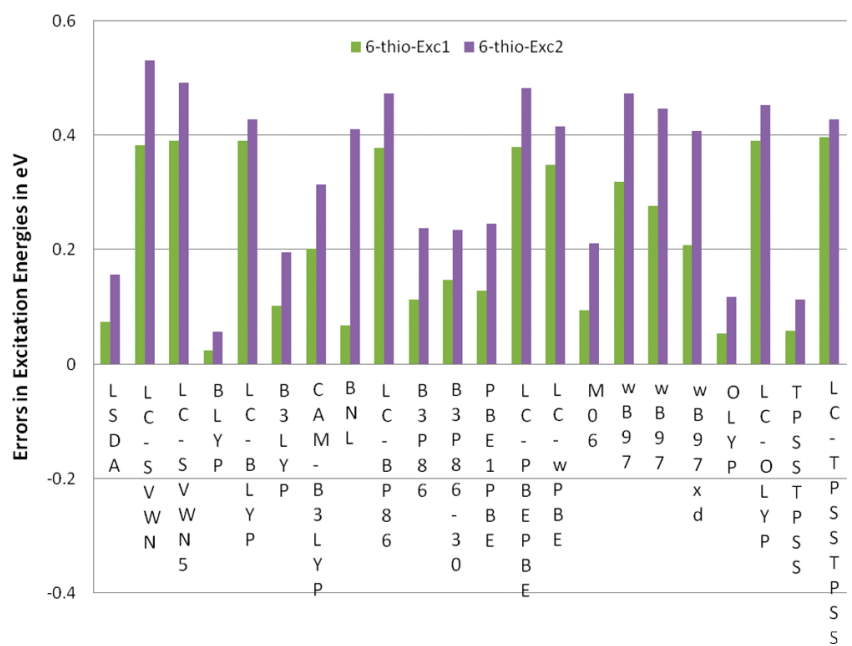

Figure 18. Errors and first and second excitation energies of $6 \mathrm{~T}^{+}$.

neutral form decreases to half its original intensity upon oxidation, while two new features appear. ${ }^{74}$

Theoretical analysis of the 2T cation spectrum shows that there are four low energy bands, two of them with negligible oscillator strengths. ${ }^{13,78}$ Double excitations contribute between 4 and $7 \%$ in the ground and excited states. ${ }^{80}$ Because the lowest energy transition of $2 \mathrm{~T}$ is not dominated by the $2-1$ transition, $2 \mathrm{~T}$ is not representative for longer oligothiophenes. For 3T to 9T, global hybrid functionals predict two bands for cations, in agreement with experimental results. ${ }^{13}$ The lower energy transition of oxidized thiophene oligomers is due to an electronic transition from HOMO to SOMO (2-1 transition) with a CI coefficient between 0.73 and 0.94 . The smaller coefficients are caused by deexcitations, and there is no correlation between $\mathrm{CI}$ coefficients and HF exchange. The second excited state is dominated by a transition from SOMO to LUMO ( $1-1^{\prime}$ transition) with a CI coefficient between 0.66 and 0.88. Again, the size of the CI coefficients appears to be random with respect to the functionals, and density functionals that predict small CI coefficients for the $1-2$ transition may predict large ones for the $1-1^{\prime}$ transition. With all functionals, there are small contributions of the $1-1^{\prime}$ transition to the $1-2$ state and vice versa. Between $8 \mathrm{~T}$ and $12 \mathrm{~T}$, the $1-2$ and $1-1^{\prime}$ transitions do not mix anymore, but the $1-1^{\prime}$ transition starts to interact with $2-2^{\prime}$ and $3-1^{\prime}$ electronic configurations and splits into two peaks. ${ }^{13}$ In agreement with experimental results, the third band of $12 \mathrm{~T}^{+}$occurs at the same position as the absorption of neutral $12 \mathrm{~T}$ at the same level of theory.

Excitation energies of $6 \mathrm{~T}$ and $6 \mathrm{~T}^{+}$are summarized in Table 2. Pure DFT and global hybrids produce $\left\langle S^{2}\right\rangle$ values that are close to the correct value of 0.75 for the ground state of $6 \mathrm{~T}^{+}$. HF predicts a value of 3.02, and long-range corrected functionals predict values between 0.85 and 1.0. Normally, a deviation of $10 \%$ is considered to be acceptable. CAM-B3LYP and wB97XD with values of 0.85 and 0.86 are therefore borderline. Values of around 1 were shown to produce useless spectra for polyene cations. ${ }^{12}$ $\left\langle S^{2}\right\rangle$ values of the first excited state are only slightly above those of the ground state, but spin contamination of the second excited state is problematic. Only pure DFT is adequate in terms of $\left\langle S^{2}\right\rangle$ for the second excited state; global hybrids are borderline. Overall, there is a pretty good correlation between $\left\langle S^{2}\right\rangle$ values and excitation energies, with larger spin contamination leading to higher excitation energy. 
Table 3. Ground state and Excited State $\left\langle S^{2}\right\rangle$ Values and Excited State Energies and Oscillator Strengths in the Absorption Spectrum of $12 \mathrm{~T}^{+}$with Different Density Functionals

\begin{tabular}{|c|c|c|c|c|c|c|c|c|c|c|}
\hline state & 0 & \multicolumn{3}{|c|}{1} & \multicolumn{3}{|c|}{2} & \multicolumn{3}{|c|}{3} \\
\hline \multirow[t]{2}{*}{ exptl74 } & \multirow[b]{2}{*}{$\left\langle S^{2}\right\rangle$} & \multicolumn{3}{|c|}{0.62} & \multicolumn{3}{|c|}{1.45} & \multicolumn{3}{|c|}{2.68} \\
\hline & & $E$ & $f$ & $\left\langle S^{2}\right\rangle$ & $E$ & $f$ & $\left\langle S^{2}\right\rangle$ & $E$ & $f$ & $\left\langle S^{2}\right\rangle$ \\
\hline BLYP & 0.75 & 0.48 & 1.02 & 0.75 & 1.11 & 1.70 & 0.83 & 1.581 .60 & 1.060 .61 & 0.810 .86 \\
\hline B3LYP & 0.77 & 0.48 & 1.53 & 0.76 & 1.35 & 1.57 & 1.44 & 1.962 .22 & 1.060 .74 & 1.940 .96 \\
\hline B3P $86-30 \%$ & 0.78 & 0.42 & 1.59 & & 1.67 & 0.81 & & 2.502 .52 & 0.481 .68 & \\
\hline CAM-B3LYP & 0.89 & 0.55 & 2.06 & 0.87 & 1.531 .80 & 1.250 .58 & 1.541 .77 & 2.873 .23 & 0.740 .57 & 1.260 .96 \\
\hline LC-BLYP & 1.10 & 0.90 & 2.29 & 1.20 & 1.551 .90 & 0.480 .75 & 2.681 .79 & 3.413 .503 .83 & 0.830 .320 .29 & 1.321 .431 .38 \\
\hline wB97XD & 0.90 & 0.62 & 1.98 & 0.90 & 1.601 .75 & 0.711 .23 & 2.271 .17 & 2.993 .043 .70 & 0.660 .390 .46 & 1.031 .010 .94 \\
\hline LC-wPBE & 1.09 & 0.84 & 2.27 & 1.18 & 1.511 .87 & 0.430 .81 & 2.701 .81 & 3.303 .403 .70 & $\begin{array}{llll}0.58 & 0.58 & 0.29\end{array}$ & 1.341 .321 .39 \\
\hline
\end{tabular}

Figure 18 shows errors in excitation energies compared to experimental values. The best agreement with experimental results is achieved with BLYP. OLYP and TPSSTPSS are also quite accurate. The more HF-exchange is used, the more the results deviate from experimental results. Long-range corrected functionals perform worse than global hybrids. Among the longrange corrected functionals, CAM-B3LYP is the best, followed by wB97XD. This is in line with the spin contamination. With all functionals, the error in the second excitation energy is larger than in the first.

The influence of HF exchange on cation spectra of $12 \mathrm{~T}^{+}$is summarized in Table 3 and illustrated in Figure 19. For these calculations, the smaller $6-31 \mathrm{G}^{*}$ basis set was used. (For $6 \mathrm{~T}^{+}$, the differences in results with $6-311 \mathrm{G}^{*}$ and $6-31 \mathrm{G}^{*}$ basis sets are about $0.1 \mathrm{eV}$.) Spin contamination does not increase from $6 \mathrm{~T}^{+}$to $12 \mathrm{~T}^{+}$for the ground state (GS) and first excited states. For excited states 2 and 3, only pure DFT produces acceptable $\left\langle S^{2}\right\rangle$ values.

The effect of HF exchange is spreading the peaks over a wider range and splitting them into several contributions. Oscillator strengths of the first peak increase while the oscillator strengths of the higher energy peaks decrease with increasing HF exchange. The vertical lines in Figure 19 at the experimental peak positions show that BLYP underestimates the peak separations and that LC-BLYP overestimates them. The agreement of peak positions with experimental results is best with CAM-B3LYP and B3P86-30\%, which seems to produce oscillator strengths that match the experimental peak heights better. The performance of pure DFT is not as good as for $6 \mathrm{~T}^{+}$. The close lying peaks are most likely a result of the too close lying orbital energies with pure DFT.

Orbital Energies. With the exact exchange-correlation functional, $-\varepsilon_{\text {HOMO }}$ should match $\mathrm{IP}_{\triangle \mathrm{SCF}}$. The BNL functional produces orbital energies that fulfill this requirement, ${ }^{20}$ but the BNL functional suffers from incorrect chain length dependence of $\mathrm{IP}_{\Delta \mathrm{SCF}}$ for extended $\pi$ systems. Because $\gamma$ is tuned to equalize $-\varepsilon_{\mathrm{HOMO}}$ with $\mathrm{IP}_{\Delta \mathrm{SCF}},-\varepsilon_{\mathrm{HOMO}}$ gets too small as well. It is not possible to find a value for $\gamma$ that simultaneously produces a correct $\mathrm{IP}_{\triangle \mathrm{SCF}}$ and a match between $\mathrm{IP}_{\triangle \mathrm{SCF}}$ and $-\varepsilon_{\mathrm{HOMO}}$ for long oligomers. ${ }^{20,81}$ LC functionals with fixed $\gamma$ were also shown to improve orbital energies of small molecules because they achieve cancellation of the Coulomb self-repulsion through the exchange self-interaction term, ${ }^{82}$ as it is known from HF theory.

Differences between $-\varepsilon_{\text {HOMO }}$ and experimental IPs are plotted in Figure 20, differences between $-\varepsilon_{\mathrm{HOMO}}$ and $\mathrm{IP}_{\Delta \mathrm{SCF}}$ in Figure 21. Figure 20 reveals that all pure DFT and global hybrid functionals grossly underestimate $-\varepsilon_{\text {HOMO IPs. Range- }}$ separated functionals lower the orbital energies, and most of them overestimate IPs. Excellent agreement with experimental results is achieved with $\mathrm{HF}$ and the wB97 series, wB97XD producing an almost perfect match. Results with CAM-B3LYP and $\mathrm{BNL}$ are acceptable.

Figure 21 shows whether $-\varepsilon_{\mathrm{HOMO}}$ IPs are consistent with $\mathrm{IP}_{\triangle \mathrm{SCF}}$ 's at a given level of theory, i.e., whether a theoretical level leads to internally consistent results without reference to experimental results. BNL is internally consistent by definition. $\mathrm{HF}$, which yields very good $-\varepsilon_{\mathrm{HOMO}}$ IPs, is internally inconsistent because the $\mathrm{IP}_{\triangle \mathrm{SCF}}$ 's are too low. Long-range corrected functionals suffer from overcorrection of the orbital energies and predict larger $-\varepsilon_{\mathrm{HOMO}}$ IPs than $\mathrm{IP}_{\triangle \mathrm{SCF}}$ 's. The best results in terms of accuracy compared to experimental results, internal consistency, and chain length dependence are achieved with wB97XD. CAM-B3LYP is acceptable.

The proof that $-\varepsilon_{\text {HOMO }}$ is equal to the first IP with the unknown exact exchange-correlation functional cannot be easily extended to other orbital energies. ${ }^{83,84}$ Therefore, controversy exists about lower lying orbitals and about unoccupied orbitals. The energies of the relevant states can be calculated by adding excitation energies of cations that create holes in the appropriate orbitals to $\mathrm{IP}_{\triangle \mathrm{SCF}}$. The idea to model photoelectron spectra in this way is described in refs 14 and 20 .

Figure 22 compares higher ionic states with negative energies of lower lying orbitals for $1 \mathrm{~T}$ at the $\mathrm{wB} 97 \mathrm{XD} / 6-311 \mathrm{G}^{*}$ level. In the valence region, there is a one to one correspondence between negative orbital energies and the corresponding states. The states arise from electronic transitions of $\beta$ electrons from lower lying orbitals into the SOMO. The contributions (CI coefficients) of these transitions are above 0.98 in the energy range from the first IP down to $15 \mathrm{eV}$. This means that these states are singleconfigurational and that the ionization is a single electron process. States with higher ionization energies than $16 \mathrm{eV}$ are multiconfigurational. Plotted in Figure 22 are all states that have a contribution from an electron configuration that has a hole in one of the $\beta$ orbitals. The lengths of the peaks are the squared CI coefficients of these electronic configurations. It can be seen that the states with holes in $\beta$ orbitals cluster around the negative energy of the corresponding orbitals. For the whole energy range from 8 to $28 \mathrm{eV}$, there is an excellent correspondence between states and orbital energies. Figure 23 shows that the match is even better with the BNL functional. 

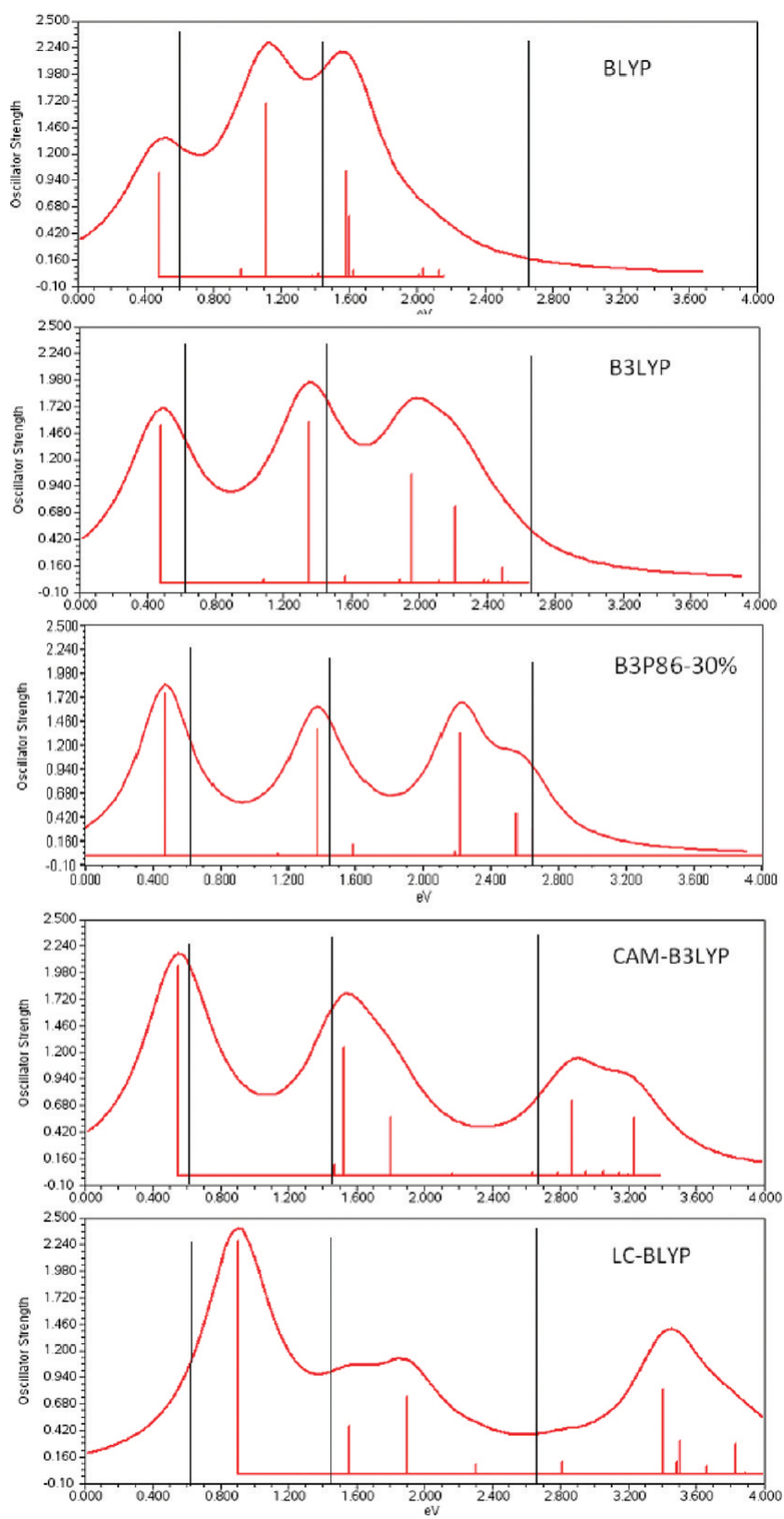

Figure 19. Spectra of $12 \mathrm{~T}^{+}$with different density functionals and the 6-31G* basis set. Experimental peak positions are shown as black vertical lines.

Figure 24 compares the $w B 97 X D / 6-311 G^{*}$ orbital energies with experimental peak positions. The excellent match of negative orbital energies with photoelectron peaks (PE) shows that the wB97XD functional is capable (like BNL) of predicting reliable orbital energies for small systems without any adjustments.

What is left to show is that the results are not deteriorating with increasing chain lengths, a problem that cannot be solved with BNL. Figure 25 compares negative orbital energies and states to PE peaks of 4T. The resolution of the experimental 4T spectrum is much less than that of $1 \mathrm{~T}$, but the overall agreement between peak position and negative orbital energies holds over the whole range of available PE peaks.

The only thiophene oligomer for which experimental EAs seem to exist is 2T. $2 \mathrm{~T}$ has a positive EA of $0.049 \pm 0.005 \mathrm{eV}$

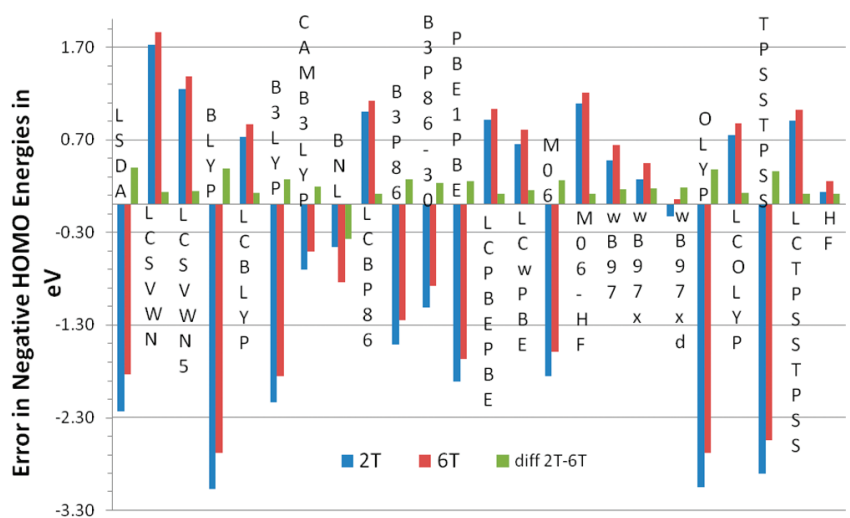

Figure 20. Difference between negative HOMO energies and experimental IPs of $2 \mathrm{~T}$ and $6 \mathrm{~T}$.

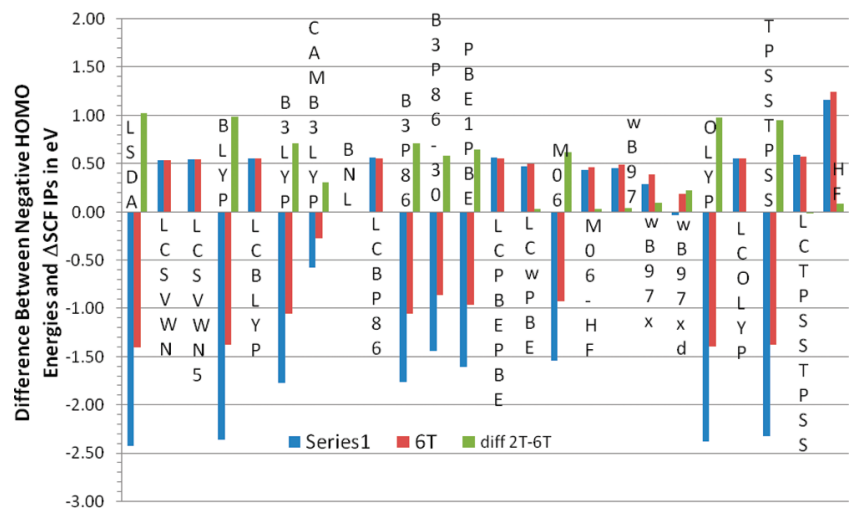

Figure 21. Difference between negative HOMO energies and $\triangle S C F$ IPs of $2 \mathrm{~T}$ and $6 \mathrm{~T}$ with different density functionals.

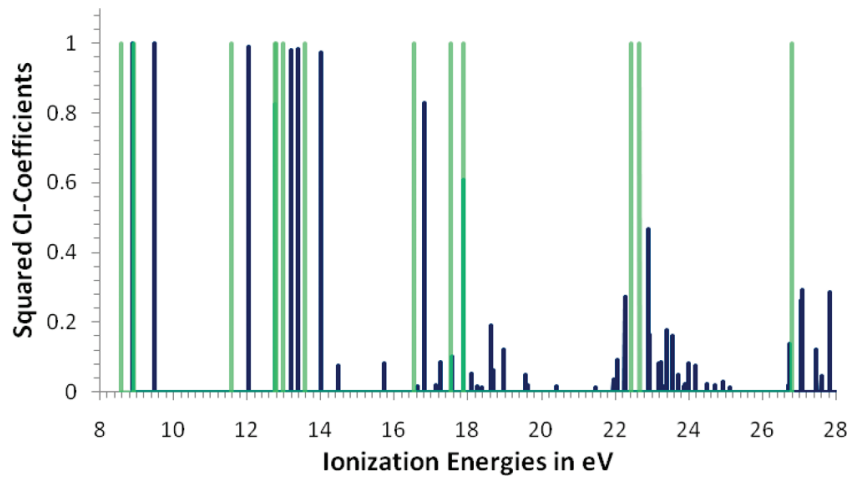

Figure 22. Ground and excited states of $1 \mathrm{~T}^{+}$(blue lines) compared to negative orbital energies (green lines) at $\mathrm{wB} 97 \mathrm{XD} / 6-311 \mathrm{G}^{*}$.

according to photoelectron spectroscopy (PES) of the anion. ${ }^{86}$ Since the electron is removed from a stable anion, the EA from the PES experiment is adiabatic. Electron transmission spectroscopy (ETS) produces an attachment energy at $0.2 \mathrm{eV} .{ }^{68}$ Attachment energies are approximate negative vertical EAs. ${ }^{68}$ Thus, $2 \mathrm{~T}^{-}$at the geometry of the neutral form is unbound according to ETS.

To evaluate the contribution of the zero point energy (ZPE), frequency calculations were carried out on $2 \mathrm{~T}$ and $2 \mathrm{~T}^{-}$at the $\mathrm{wB} 97 \mathrm{XD} / 6-311+\mathrm{G}^{*}$ level. The ZPE correction for the EA of $2 \mathrm{~T}$ is 


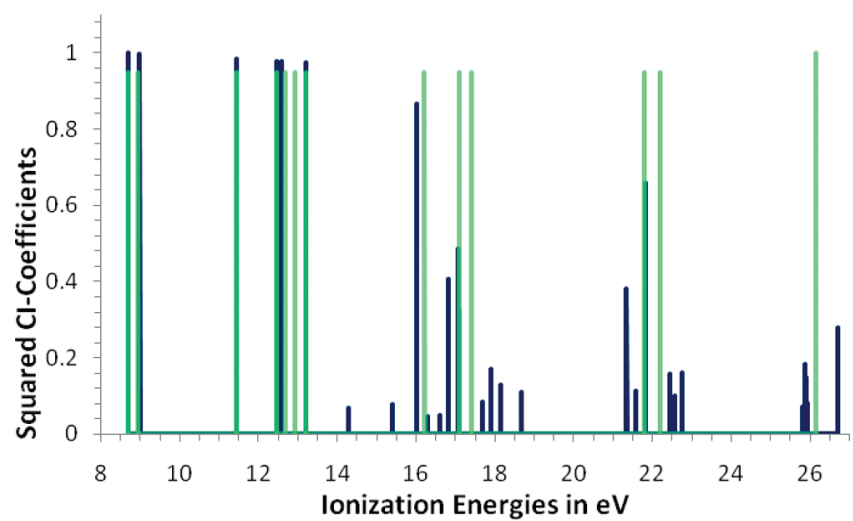

Figure 23. Ground and excited states of $1 \mathrm{~T}^{+}$(blue lines) compared to negative orbital energies (green lines) at BNL/6-311G*.

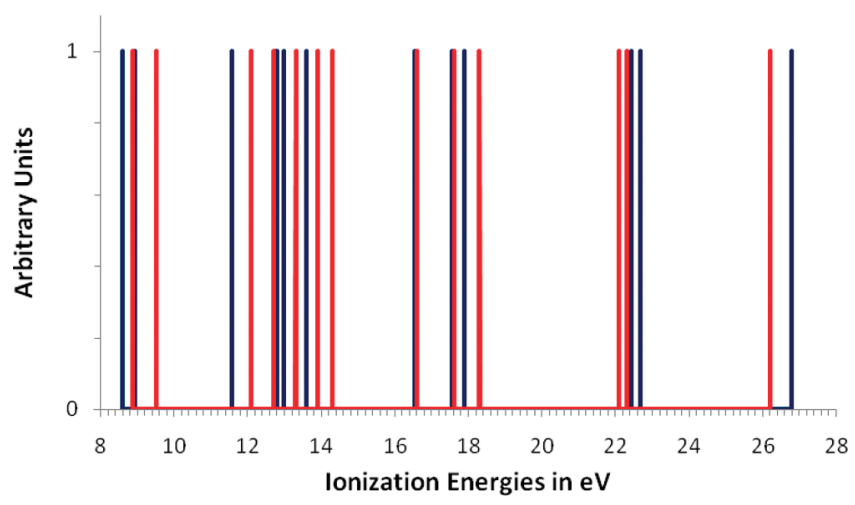

Figure 24. Negative orbital energies (blue lines) at wB97XD/6-311G and experimental ${ }^{85} \mathrm{PE}$ peaks (red lines) of $1 \mathrm{~T}$.

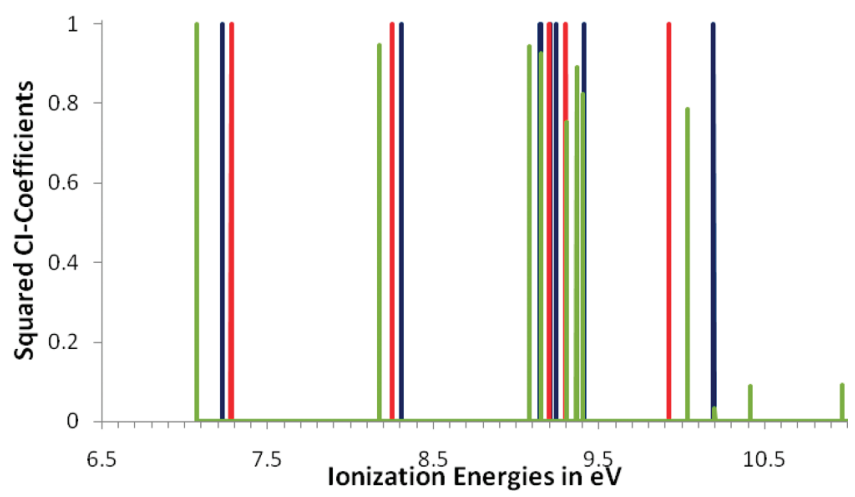

Figure 25. Negative orbital energies (blue lines), states (green lines) at wB97XD/6-311G* and experimental ${ }^{85} \mathrm{PE}$ peaks (red lines) of 4T.

$0.135 \mathrm{eV}$. As this value is larger than the experimental adiabatic EA, $2 \mathrm{~T}^{-}$is unbound in its equilibrium structure before $\mathrm{ZPE}$ correction. Orbital energies of neutral species correspond to vertical EAs, and a negative EA of $2 \mathrm{~T}$ indicates that LUMO energy, if correct, must be positive. There seem to be no PES experiments on longer oligomers, and ETS data of longer oligomers do not simply correlate with orbital energies from semiempirical calculations. ${ }^{68}$ Therefore, only the vertical EA of $2 \mathrm{~T}$ is compared with experimental values, and $6 \mathrm{~T}$ is checked for internal consistency.

Because vertical and adiabatic EAs of $2 \mathrm{~T}$ are negative before ZPE correction, the electron would simply be removed from the

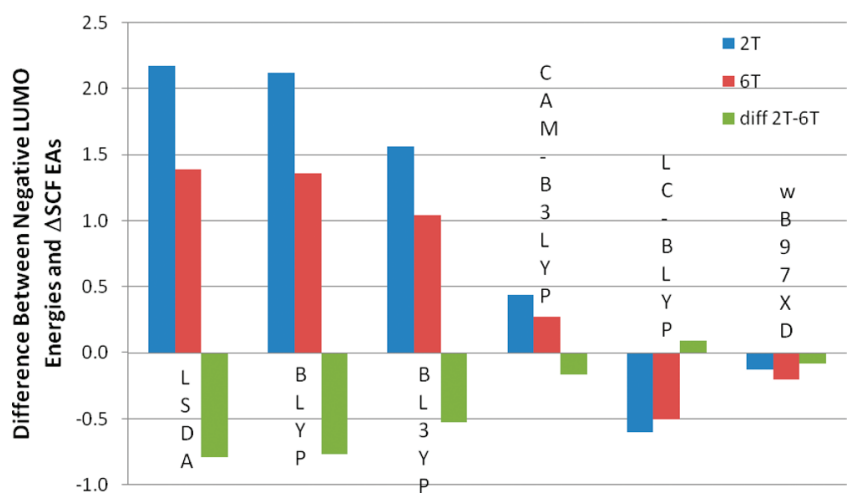

Figure 26. Difference between negative LUMO energies and $\triangle$ SCF EAs of $2 \mathrm{~T}$ and $6 \mathrm{~T}$ with different density functionals.

$2 \mathrm{~T}$ molecule with an infinite basis set. With finite basis sets, the electron is forced to stay on the anion, and an attempt can be made to estimate the energy difference between $2 \mathrm{~T}$ and $2 \mathrm{~T}^{-}$. At the CCSD/aug-CCPVTZ level of theory, the vertical EA of $2 \mathrm{~T}$ is $-0.428 \mathrm{eV}$ with $\mathrm{ZPE}$ correction, which is reasonably close to the ETS result of $-0.2 \mathrm{eV}$. We were unable to include approximate triples with the aug-CCPVTZ basis set, but with the 6-311G* basis set, approximate triples contribute only $0.019 \mathrm{eV}$. Density functional theory with the exception of LSDA predicts negative EAs as well. The values of the vertical EAs including the ZPE are LSDA, $0.476 \mathrm{eV}$; BLYP, $-0.224 \mathrm{eV}$; B3LYP, $-0.203 \mathrm{eV}$; CAMBLYP, $-0.367 \mathrm{eV}$; LC-BLYP, $-0.482 \mathrm{eV}$; wB97XD, $-0.444 \mathrm{eV}$ with the $6-311 \mathrm{G}^{*}$ basis set. Inclusion of diffuse functions with $6-311+G^{*}$ and aug-CCPVTZ basis sets increases the EA by $0.2-0.3 \mathrm{eV}$.

Figure 26 shows the difference between vertical $\triangle$ SCF EAs and LUMO energies of $2 \mathrm{~T}$ and $6 \mathrm{~T}$ with six different density functionals. All levels of theory predict $6 \mathrm{~T}^{-}$to be stable. LSDA and BLYP have LUMO orbitals that are lying too low and predict therefore EAs that are significantly larger than the $\triangle$ SCF EAs. With global hybrids, i.e., B3LYP, the LUMO is pushed up and the difference between negative LUMO energies and EAs decreases. Long-range correction fixes this problem to a large extent, but the agreement differs from functional to functional. CAM-B3LYP does not correct enough, and LC-BLYP overcorrects. The differences between orbital energies and EAs are larger for $2 \mathrm{~T}$ than for 6T. wB97XD leads to a near perfect match for 2T and 6T between negative LUMO energy and EA. These findings are very similar to those for IPs.

\section{DISCUSSION}

Performance of Range-Separated Functionals. Taking all results together and setting the maximal allowable error for any property of any system to $0.5 \mathrm{eV}$, only the $\mathrm{wB} 97 \mathrm{XD}$ functional emerges as sufficient. The largest errors with wB97XD are for excited states of cations. Second best is the performance of CAMB3LYP, which is superior in excitation energies of cations but exceeds errors of $0.5 \mathrm{eV}$ for orbital energies. M06 and TPSSTPSS do not have any obvious advantages for conjugated $\pi$ systems. All range-separated functionals are capable of correcting the incorrect chain length dependence of IPs, $E_{\mathrm{g}}$ 's, and orbital energies that make problems with pure DFT and global hybrids. Here, CAM-B3LYP, which contains only $65 \%$ long-range HF exchange and has therefore an incorrect long-range limit, tends to be 
slightly inferior. Bond length data, IPs and $E_{\mathrm{g}}$ 's show, however, that the LC correction tends to overcorrect and approaches HF results too closely. Therefore, BLA and nonplanarity are somewhat overestimated, and defect sizes may be underestimated with the LC functionals which have no short-range HF exchange and as a result an earlier onset of $100 \%$ HF-exchange (larger $\gamma$ ). Thus, range-separated functionals that include a small amount of shortrange HF exchange perform decidedly better for conjugated $\pi$ systems than those without.

A comparison of thiophene and furan oligomers indicates that there are differences between systems. Properties of furan oligomers require more HF exchange and are better predicted with LC functionals than those of thiophenes, although a tendency to overshoot is also predicted here. For furans, CAM-B3LYP might not be enough, and wB97XD appears to be a good compromise. The larger error for the thiophene compared to furan oligomers with LC functionals might be due in part to the overestimated nonplanarity.

Caution has to be applied when cations or anions are to be investigated. Range-separated hybrid functionals are more likely to produce spin-contaminated wave functions than global hybrids. This causes inaccuracies in the higher energy peaks in spectra of doped species. Here, LC functionals overshoot clearly and CAM-B3LYP and $\mathrm{wB} 97 \mathrm{XD}$ are more suitable. In particular, because of the excellent match between its negative orbital energies and states, wB97XD is the best choice overall.

The difference between the LC-corrected GGA functionals and CAM-B3LYP, wB97X, and wB97XD is that the latter contain short-range HF-exchange and a larger short range (smaller $\gamma$ ) than the LC functionals. In this respect, the $\mathrm{wB} 97$ functional resembles the LC functionals, but it produces generally smaller errors than any of them. Therefore, the superior performance of wB97X and wB97XD seems to be due to a suitable parametrization of the basic functional plus a proper amount of short-range HF exchange in combination with an adequate size of the short range. It is especially encouraging that the functional performs extremely well on properties like excitation energies and orbital energies, which were not included in the training set for the parametrization.

Defect Sizes and Shapes. With range-separated hybrid functionals, singly charged defects shrink and converge to a size of about 9 or 10 rings or $36-40$ carbon atoms for polyenes, thiophenes, and furans. As mentioned above, defect sizes approach those at the HF level (30 atoms) and are probably underestimated. In dications, two clearly separated defects at the chain ends form that extend also over about $9-$ or 10 rings. The chain segment between the two defects is completely undistorted. Range-separated hybrid functionals were shown to correct the error of pure DFT and global hybrids with the wrong dissociation limit of symmetrical radical cations. ${ }^{49}$ Therefore, a crucial error of DFT is eliminated. This error might have influenced results with global hybrids regarding the nonexistence of bound bipolarons. The pronounced separation of two positive charges into two defects located at the ends of the chains confirms therefore that there is no bipolaron binding energy in conducting organic polymers.

Orbital Energies, Transport Gap, and Optical Band Gap. In principle, there are three band gaps: $E_{\mathrm{g}}$, which is the first peak in the UV spectrum (either the onset of absorption or $\varepsilon_{\max }$ ); the electrochemical band gap $E_{\mathrm{el}}$, which is the difference between oxidation and reduction potential; and $E_{\mathrm{t}}$, which is the difference between IP and EA. Although the three band gaps are obviously
Table 4. IPs, EAs, and Energy Gaps of Isolated 6T and a Cluster of Five 6T Chains in $\mathrm{eV}$ with the wB97XD Functional

\begin{tabular}{|c|c|c|c|c|c|}
\hline \multirow[t]{2}{*}{ property } & \multicolumn{2}{|c|}{$6 \mathrm{~T}$} & \multirow[b]{2}{*}{$6-31 \mathrm{G}^{*} \mathrm{pl}$} & \multirow{2}{*}{$\frac{5 \times 6 \mathrm{~T}}{6-31 \mathrm{G}^{*}}$} & \multirow{2}{*}{$\frac{\text { diff }_{\text {solid-gas }}}{6-31 G^{*}}$} \\
\hline & $6-311 G^{*}$ & $6-31 \mathrm{G}^{*}$ & & & \\
\hline $\mathrm{IP}_{\Delta \mathrm{SCF}}($ vert $)$ & 6.85 & 6.61 & 6.44 & 5.73 & -0.88 \\
\hline$-\varepsilon_{\text {НОМо }}$ & 7.04 & 6.80 & 6.64 & 6.01 & -0.79 \\
\hline $\mathrm{IP}_{\Delta \mathrm{SCF}}(\mathrm{ad})$ & 6.44 & 6.23 & 6.19 & 5.24 & -0.99 \\
\hline $\mathrm{EA}_{\triangle \mathrm{SCF}}($ vert $)$ & 0.61 & 0.51 & 0.80 & 0.92 & 0.41 \\
\hline$-\varepsilon_{\text {LUMO }}$ & 0.41 & 0.31 & 0.50 & 0.60 & 0.29 \\
\hline $\mathrm{EA}_{\Delta \mathrm{SCF}}(\mathrm{ad})$ & 1.03 & 0.88 & 0.94 & 1.37 & 0.49 \\
\hline$E_{\mathrm{t}}($ vert $)$ & 6.24 & 6.10 & 5.64 & 4.81 & -1.29 \\
\hline$E_{\mathrm{H}-\mathrm{L}}$ & 6.63 & 6.49 & 6.14 & 5.41 & -1.08 \\
\hline$E_{\mathrm{t}}(\mathrm{ad})$ & 5.41 & 5.35 & 5.25 & 3.87 & -1.48 \\
\hline$E_{\mathrm{g}}$ & 3.41 & 3.30 & 3.03 & 3.55 & 0.25 \\
\hline
\end{tabular}
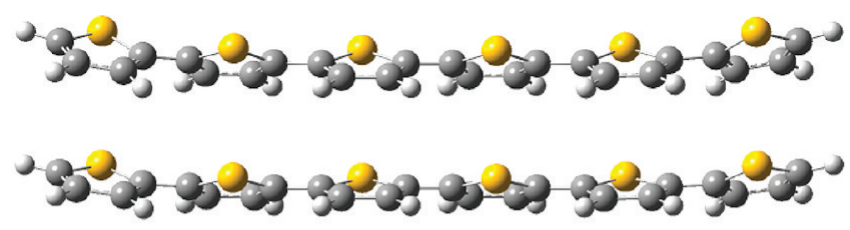

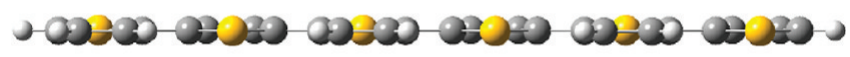

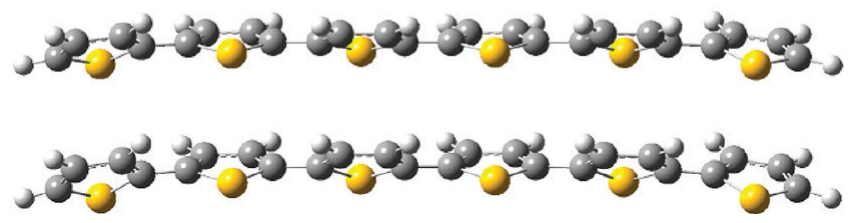

Figure 27. Five molecules of $6 \mathrm{~T}$ stacked parallel; neutral form optimized at $\mathrm{wB} 97 \mathrm{XD} / 6-31 \mathrm{G}^{*}$.

correlated, the exact relationship between them is rather complicated. ${ }^{87,88}$ In experiments on thin films, $E_{\mathrm{g}}$ and $E_{\mathrm{el}}$ are often quite close, differing by several tenths of an electronvolt. From a conceptual point of view, $E_{\mathrm{el}}$ and $E_{\mathrm{t}}$ might be expected to be closest because both correspond to the difference between independent electron removal and electron attachment energies, while electron and hole are not completely separated in the UV experiment. The relationship between $E_{\mathrm{t}}$ and $E_{\mathrm{g}}$ has been investigated with PES, inverse PES (IPES), and UV spectroscopy of thin films. ${ }^{88}$ The directly measured difference between IP and EA of $6 \mathrm{~T}$ is $4.2 \mathrm{eV} . E_{\mathrm{g}}$ is $3.0 \mathrm{eV}$. After applying a range of corrections for polarization, relaxation, peak centers, difference between surface and bulk, and so on, the difference between $E_{\mathrm{t}}$ and $E_{\mathrm{g}}$ was estimated to be $0.4 \mathrm{eV} .^{88}$

In gas-phase calculations with global hybrid functionals, there is a relatively close match of $E_{\mathrm{g}}$ and $E_{\mathrm{H}-\mathrm{L}} \cdot{ }^{18,28,30}$ Because accurate negative orbital energies formally match IPs and EAs, $E_{\mathrm{H}-\mathrm{L}}$ should match $E_{\mathrm{t}}$ rather than $E_{\mathrm{g}} \cdot{ }^{87,88}$ Given the small experimental difference between the two and the considerable uncertainty in the DFT orbital energies, the agreement was usually found to be sufficient. Having now reliable orbital energies at the wB97XD level allows us to address this issue more thoroughly. Vertical and adiabatic IPs and EAs of 6T are collected in Table 4. The $E_{\mathrm{t}}$ of $6 \mathrm{~T}$ 


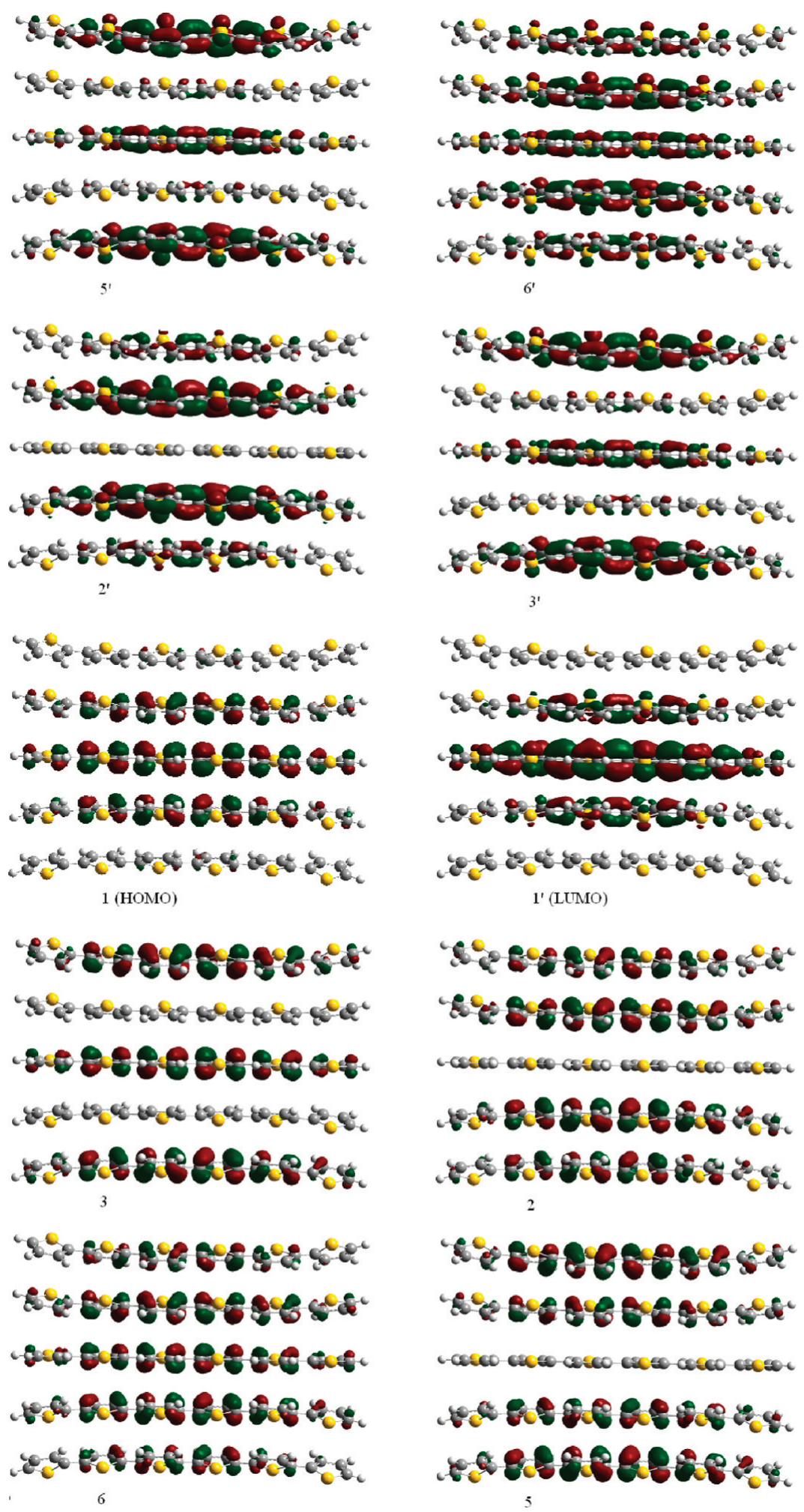

Figure 28. Molecular orbitals of a $\pi$-stacked cluster of five $6 \mathrm{~T}$ molecules.

in the gas phase amounts to $6.24 \mathrm{eV}$. This is matched fairly well by the HOMO-LUMO gap $\left(E_{\mathrm{H}-\mathrm{L}}\right)$ of $6.63 \mathrm{eV}$. $E_{\mathrm{g}}$ calculated with TDwB97XD/6-311G* is $3.41 \mathrm{eV}$. Thus, the difference between $E_{\mathrm{t}}$ and $E_{\mathrm{g}}$ is $2.83 \mathrm{eV}$ for $6 \mathrm{~T}$ in the gas phase. For comparison, B3LYP yields $E_{\mathrm{t}}=6.18 \mathrm{eV}-1.23 \mathrm{eV}=4.95 \mathrm{eV}$. This value is too small because of the incorrect chain length dependence of $\mathrm{IP}_{\triangle \mathrm{SCF}}$ and $\mathrm{EA}_{\triangle \mathrm{SCF}} \cdot E_{\mathrm{H}-\mathrm{L}}=5.12 \mathrm{eV}-2.27$ $\mathrm{eV}=2.85 \mathrm{eV}$, which is even smaller because of the incorrect orbital energies with global hybrids. $E_{\mathrm{g}}=2.56 \mathrm{eV}$, which is underestimated (by $0.36 \mathrm{eV}$ ) because of incorrect chain length dependence. The good match between $E_{\mathrm{H}-\mathrm{L}}$ and $E_{\mathrm{g}}$ at B3LYP is thus a coincidence. 


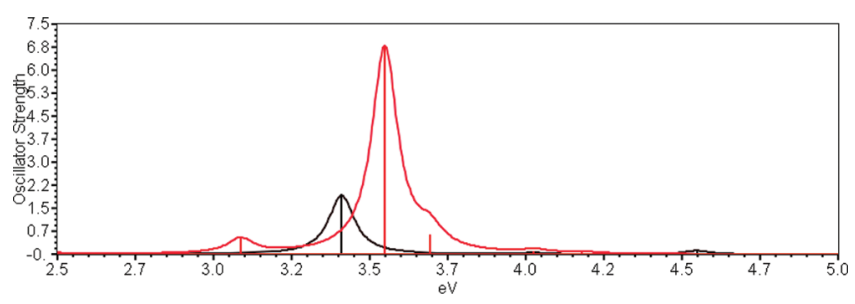

Figure 29. UV spectra of 6T (black) and a cluster of five 6T molecules (red).

Because the wB97XD gas phase values were shown to be reliable throughout this paper, the large discrepancy between the theoretical $(2.83 \mathrm{eV})$ and experimental $(0.4 \mathrm{eV})$ differences between $E_{\mathrm{t}}$ and $E_{\mathrm{g}}$ can only be caused by solid state effects. In order to investigate the influence of neighboring chains, we optimized a cluster of five 6T chains (Figure 27) in neutral, positively, and negatively charged states at the wB97XD/6-31G* level and calculated IPs and EAs. The results are included in Table 4. Although a small $\pi$-stacked cluster is a very crude model for a herringbone structured crystal of $6 \mathrm{~T}$, we believe that the model catches the essentials at least qualitatively. Since wB97XD includes dispersion, the geometry optimization yielded a reasonable distance between the chains of $4.5 \AA$. The spacing between chains in the cation is $3.4 \AA$; in the anion it is $3.6 \AA$. In agreement with the experimental crystal structures of $6 \mathrm{~T}$ and $8 \mathrm{~T}$, the inner $6 \mathrm{~T}$ is planar.

Vertical IP, adiabatic IP, and $-\varepsilon_{\text {HOMO }}$ decrease from the gas phase to cluster by between 0.8 and $1.0 \mathrm{eV}$. The electron affinitiy increases by $0.3-0.5 \mathrm{eV}$. Thus, the transport gap is reduced by $1.1-1.5 \mathrm{eV}$ in the cluster. About $0.5 \mathrm{eV}$ of that is due to planarization. The first excitation energy of the cluster is $0.25 \mathrm{eV}$ higher than that of 6T. Theory therefore predicts that the difference between $E_{\mathrm{t}}$ (vert) and $E_{\mathrm{g}}$ shrinks to $1.26 \mathrm{eV}$ in the cluster. For comparison with experimental results, we need to consider furthermore that the solid state IPs and EAs are adiabatic. ${ }^{88}$ For the cluster, we should therefore compare $E_{\mathrm{t}}(\mathrm{ad})$ to $E_{\mathrm{g}}$. This difference is $0.32 \mathrm{eV}$, in very good agreement with the experimental value of $0.4 \mathrm{eV} .^{88} E_{\mathrm{H}-\mathrm{L}}$, albeit $1.08 \mathrm{eV}$ smaller in the cluster than in the gas phase, overestimates $E_{\mathrm{g}}$ of the cluster by $1.86 \mathrm{eV}$ mainly because it approximates $E_{\mathrm{t}}(\mathrm{vert})$ rather than $E_{\mathrm{t}}(\mathrm{ad})$.

The opposite effect of neighboring chains on $E_{\mathrm{t}}$ and $E_{\mathrm{g}}$ is caused on the one hand by the fact that HOMO and LUMO delocalize to some extent over the whole structure (Figure 28), which lowers the IP and increases the EA. On the other hand, $E_{\mathrm{g}}$ increases because the HOMO-LUMO transition is symmetryforbidden in the cluster. The lowest allowed state involves transitions between higher and lower lying orbitals, namely, $6-1^{\prime}, 5-2^{\prime}, 3-3^{\prime}, 2-5^{\prime}$, and $1-6^{\prime}$. The spectra of $6 \mathrm{~T}$ and the cluster are shown in Figure 29. Both spectra have one main feature at similar energies, but the cluster peak has a much larger oscillator strength. These findings rationalize the puzzling observation that there is a large solid state polarization energy for IPs of organic solids $(\sim 1.8 \mathrm{eV})^{89}$ but very little difference between UV absorption energies of organic molecules in the gas phase, in solution, and in the solid state. Our results furthermore predict that the solid state polarization energy is larger for IPs than for EAs, in contrast to earlier assumptions. ${ }^{88}$

\section{CONCLUSIONS}

Range-separated functionals solve the DFT problem of incorrect chain length dependence of IPs, excitation energies, and orbital energies of conjugated systems. Using 100\% of long-range HF exchange can overcorrect, however, as is obvious from the results on BLA, twist angles, and orbital energies. Of all rangeseparated functionals tested, wB97X, wB97XD, and CAMB3LYP show the best overall performance, especially when excited states of cations are included. The reason seems to be that these functionals include short-range HF exchange and have a larger short range. This pushes the onset of $100 \%$ HF exchange further out and leads to less overshooting.

Because range-separated hybrid functionals correct the erroneous dissociation of symmetrical radical cations, a major error of approximate DFT that might have had an impact on the nature of the charge carriers in conducting polymers has been removed. The fact that range-separated functionals not only confirm but enhance the splitting of two charges on one chain into two separated defects provides further support to our earlier claim ${ }^{13}$ that there are no bound bipolarons in conducting polymers.

Among all functionals, wB97XD appears most promising for calculations on conducting polymers. In particular, the excellent match of negative orbital energies (with binding energies of up to $30 \mathrm{eV}$ ) and IPs and EAs makes it useful for band structure calculations. Only the higher excited states of charged systems are somewhat problematic.

The good accuracy of wB97XD orbital energies allows assessment of the relationship between $E_{\mathrm{H}-\mathrm{L}}, E_{\mathrm{g}}$, and $E_{\mathrm{t}}$ in the gas phase and in a cluster as a model for the solid state. $E_{\mathrm{H}-\mathrm{L}}$ approximates $E_{\mathrm{t}}$ (vert) with errors of 0.4 and $0.6 \mathrm{eV}$ in the gas phase and in the cluster, respectively. $E_{\mathrm{t}}$ (vert) exceeds $E_{\mathrm{g}}$ by $2.8 \mathrm{eV}$ in the gas phase and by $1.26 \mathrm{eV}$ in the cluster. As IPs and EAs of thin films are adiabatic, for the cluster, $E_{\mathrm{g}}$ has to be compared to $E_{\mathrm{t}}(\mathrm{ad})$, and the difference is only $0.32 \mathrm{eV}$. Nonetheless, even if $E_{\mathrm{g}}$ is dominated by a HOMO-LUMO transition, which may or may not be the case, it is not equal to $E_{\mathrm{H}-\mathrm{L}}$. Therefore, correct orbital energy differences do not predict optical band gaps, and the "success" of DFT orbital energies with global hybrids to do so is a result of error cancellation between incorrect orbital energies, wrong chain length dependence, and the neglect of solid state polarization effects.

\section{AUTHOR INFORMATION}

\section{Corresponding Author}

*Tel.: +90 312290 2122. Fax: +90 312266 4068. E-mail: salzner@fen.bilkent.edu.tr.

\section{ACKNOWLEDGMENT}

This work was supported by TUBITAK, Grant TBAG 109 T426.

\section{REFERENCES}

(1) Su, W. P.; Schrieffer, J. R.; Heeger, A. J. Phys. Rev. B 1980, 22, 2099-2111.

(2) Bertho, D.; Jouanin, C. Phys. Rev. B 1987, 35, 626-633.

(3) Brédas, J.-L. In Handbook of Conducting Polymers; Skotheim, T. A., Ed.; Dekker: New York, 1986; pp 859-913.

(4) Brédas, J. L.; Street, G. B. Acc. Chem. Res. 1985, 18, 309-315.

(5) Wendel, H.; Otto, P.; Seel, M.; Ladik, J. Solid State Commun. 1985, 54, 551-554.

(6) Otto, P.; Bakhshi, A. K.; Ladik, J. THEOCHEM 1986, 135, 209-223.

(7) Otto, P.; Ladik, J. Synth. Met. 1990, 36, 327-335.

(8) Villar, H.; Otto, P.; Dupuis, M.; Ladik, J. Synth. Met. 1993, $59,97-110$ 
(9) Villar, H. O.; Otto, P.; Dupuis, M.; Ladik, J. Synth. Met. 1993, $59,97-110$.

(10) Brocks, G. J. Phys. Chem. 1996, 100, 17327-17333.

(11) Vogl, P.; Campbell, C. K. Phys. Rev. Lett. 1989, 62, 2012-2015.

(12) Salzner, U. J. Chem. Theory Comput. 2007, 3, 219-231.

(13) Salzner, U. J. Chem. Theory Comput. 2007, 3, 1143-1157.

(14) Salzner, U. J. Phys. Chem. A 2010, 114, 10997-11007.

(15) Cai, Z.-L.; Sendt, K.; Reimers, J. R. J. Chem. Phys. 2002, $117,5543-5549$.

(16) Williams, A. R; von Barth, U. In Theory of the Inhomogeneous Electron Gas; Lundqvist, S., March, N. H., Eds.; Plenum Press: London, 1983.

(17) Perdew, J. P.; Levy, M. Phys. Rev. B 1997, 56, 16021.

(18) Salzner, U.; Lagowski, J. B.; Pickup, P. G.; Poirier, R. A. J. Comput. Chem. 1997, 18, 1943-1953.

(19) Chong, D. P.; Gritsenko, O. V.; Baerends, E. J. J. Chem. Phys. 2002, 116, 1760-1772.

(20) Salzner, U.; Baer, R. J. Chem. Phys. 2009, 131, 231101-4.

(21) Stowasser, R.; Hoffmann, R. J. Am. Chem. Soc. 1999, 121, 3414-3420.

(22) Jacquemin, D.; Adamo, C. J. Chem. Theory Comput. 2011, 7, 369-376.

(23) Choi, C. H.; Kertesz, M.; Karpfen, A. J. Chem. Phys. 1997, 107, 6712-6721.

(24) Champagne, B.; Spassova, M. Phys. Chem. Chem. Phys. 2004, 6, 3167-3174.

(25) Salzner, U. J. Phys. Chem. A 2008, 112, 5458-5466.

(26) Zade, S. S.; Bendikov, M. J. Phys. Chem. B 2006, 110, 15839-15846.

(27) Zamoshchik, N.; Salzner, U.; Bendikov, M. J. Phys. Chem. C 2008, 112, 8408-8418.

(28) Salzner, U.; Pickup, P. G.; Poirier, R. A.; Lagowski, J. B. J. Phys. Chem. A 1998, 102, 2572-2578.

(29) Körzdörfer, T.; Kümmel, S.; Marom, N.; Kronik, L. Phys. Rev. B 2009, 79, 201205-4.

(30) Zade, S. S.; Zamoshchik, N.; Bendikov, M. Acc. Chem. Res. 2011, $44,14-24$

(31) Savin, A. In Recent Advances in Density Functional Methods Part I; Chong, D. P., Ed.; World Scientific: Singapore, 1995; pp 129.

(32) Iikura, H.; Tsuneda, T.; Yanai, T.; Hirao, K. J. Chem. Phys. 2001, $115,3540-3544$.

(33) Kamiya, M.; Tsuneda, T.; Hirao, K. J. Chem. Phys. 2002, $117,6010-6015$.

(34) Jaramillo, J.; Scuseria, G. E.; Ernzerhof, M. J. Chem. Phys. 2003, $118,1068-1073$.

(35) Tawada, Y.; Tsuneda, T.; Yanagisawa, S.; Yanai, T.; Hirao, K. J. Chem. Phys. 2004, 120, 8425-8433.

(36) Toulouse, J.; Colonna, F.; Savin, A. Phys. Rev. A 2004, 70, 062505 .

(37) Baer, R.; Neuhauser, D. Phys. Rev. Lett. 2005, 94, 43002.

(38) Kamiya, M.; Sekino, H.; Tsuneda, T.; Hirao, K. J. Chem. Phys. 2005, 122, 234111-10.

(39) Sato, T.; Tsuneda, T.; Hirao, K. J. Chem. Phys. 2005, 123, 104307-10.

(40) Sato, T.; Tsuneda, T.; Hirao, K. Mol. Phys. 2005, 103, 1151-1164.

(41) Sekino, H.; Maeda, Y.; Kamiya, M. Mol. Phys. 2005, 103, 2183-2189.

(42) Vydrov, O.; Heyd, J.; Krukau, A. V.; Scuseria, G. E. J. Chem. Phys. 2006, 125, 074106-1-9.

(43) Livshits, E.; Baer, R. Phys. Chem. Chem. Phys. 2007, 9, 2932-2941.

(44) Salzner, U. J. Phys. Chem. B 2003, 107, 1129-1134.

(45) Gidron, O.; Diskin-Posner, Y.; Bendikov, M. J. Am. Chem. Soc. 2010, 132, 2148-2150.

(46) Samdal, S.; Samuelsen, E. J.; Volden, H. V. Synth. Met. 1993, 59, 259.

(47) Frisch, M. J.; Trucks, G. W.; Schlegel, H. B.; Scuseria, G. E.; Robb, M. A.; Cheeseman, J. R.; Scalmani, G.; Barone, V.; Mennucci, B.;
Petersson, G. A.; Nakatsuji, H.; Caricato, M.; Hratchian, H. P.; Izmaylov, A. F.; Bloino, J.; Zheng, G.; Sonneberg, J. L.; Hada, M.; Ehara, M.; Toyota, K.; Fukuda, R.; Hasegawa, J.; Ishida, M.; Nakajima, T.; Honda, Y.; Kitao, O.; Nakai, H.; Vreven, T.; Montgomery, J. A., Jr.; Peralta, J. E.; Ogliaro, F.; Bearpark, M.; Heyd, J. J.; Brothers, E.; Kudin, K. N.; Staroverov, V. N.; Kobayashi, R.; Normand, J.; Raghavachari, K.; Rendell, A.; Burant, J. C.; Iyengar, S. S.; Tomasi, J.; Cossi, M.; Rega, N.; Millam, J. M.; Klene, M.; Knox, J. E.; Cross, J. B.; Bakken, V.; Adamo, C.; Jaramillo, J.; Gomperts, R.; Stratmann, R. E.; Yazyev, O.; Austin, A. J.; Cammi, R.; Pomelli, C.; Ochterski, J. W.; Martin, R. L.; Morokuma, K.; Zakrzewski, V. G.; Voth, G. A.; Salvador, P.; Dannenberg, J. J.; Dapprich, S.; Daniels, A. D.; Farkas, O.; Foresman, J. B.; Ortiz, J. V.; Cioslowski, J.; Fox, D. J. Gaussian 09; Gaussian, Inc.: Wallinford, CT, 2009.

(48) Shao, Y.; et al. Phys. Chem. Chem. Phys. 2006, 8, 3172-3191.

(49) Vydrov, O. A.; Scuseria, G. E. J. Chem. Phys. 2006, 125, 234109-9.

(50) Tao, J. M.; Perdew, J. P.; Staroverov, V. N.; Scuseria, G. E. Phys. Rev. Lett. 2003, 91, 146401.

(51) Vydrov, O. A.; Scuseria, G. E.; Perdew, J. P. J. Chem. Phys. 2007, 126, 154109-9.

(52) Slater, J. C. In Quantum Theory of Molecular and Solids; McGraw-Hill: New York, 1974; Vol.4.

(53) Vosko, S. H.; Wilk, L.; Nusair, M. Can. J. Phys. 1980, $58,1200-1211$.

(54) Becke, A. D. Phys. Rev. A 1988, 38, 3098-3100.

(55) Lee, C.; Yang, W.; Parr, R. G. Phys. Rev. B 1988, 37, 785-789.

(56) Perdew, J. P. Phys. Rev. B 1986, 33, 8822-8824.

(57) Perdew, J. P.; Burke, K.; Ernzerhof, M. Phys. Rev. Lett. 1996, $77,3865$.

(58) Perdew, J. P.; Burke, K.; Ernzerhof, M. Phys. Rev. Lett. 1997, $78,1396$.

(59) Handy, N. C.; Cohen, A. J. Mol. Phys. 2001, 99, 403-412.

(60) Becke, A. D. J. Chem. Phys. 1993, 98, 5648-5652.

(61) Adamo, C.; Barone, V. J. Chem. Phys. 1999, 110, 6158-6170.

(62) Chai, J.-D.; Head-Gordon, M. J. Chem. Phys. 2008, 128, 084106-15.

(63) Becke, A. D. J. Chem. Phys. 1997, 107, 8554-8560.

(64) Yanai, T.; Tew, D. P.; Handy, N. C. Chem. Phys. Lett. 2004, $393,51-57$.

(65) Chai, J.-D.; Head-Gordon, M. Phys. Chem. Chem. Phys. 2008, 10, 6615-6620.

(66) Horowitz, G.; Bachet, B.; Yassar, A.; Lang, P.; Demanze, F.; Fave, J.-L.; Garnier, F. Chem. Mater. 1995, 7, 1337-1341.

(67) Fichou, D.; Bachet, B.; Demanze, F.; Billy, I.; Horowitz, G.; Garnier, F. Adv. Mater. 1996, 8, 500-504.

(68) Jones, D.; Guerra, M.; Favaretto, L.; Modelli, A.; Fabrizio, M.; Distefano, G. J. Phys. Chem. 1990, 94, 5761-5766.

(69) da Silva Filho, D. A.; Coropceanu, V.; Fichou, D.; Gruhn, N. E.; Bill, T. G.; Gierschner, J.; Cornil, J.; Bredas, J.-L. Philos. Trans. R. Soc. London, Ser. A 2007, 365, 1435-1452.

(70) Distefano, G.; Jones, D.; Guerra, M.; Favaretto, L.; Modelli, A.; Mengoli, G. J. Phys. Chem. 1991, 95, 9746-9753.

(71) Becker, R. S.; Seixas de Melo, J.; MaÃßanita, A. n. L.; Elisei, F. J. Phys. Chem. 1996, 100, 18683-18695.

(72) Colditz, R.; Grebner, D.; Helbig, M.; Rentsch, S. Chem. Phys. 1995, 201, 309-320.

(73) Andrzejak, M.; Witek, H. Theor. Chem. Acc. 2011, 129, $161-172$.

(74) van Haare, J. A. E. H.; Havinga, E. E.; van Dongen, J. L. J.; Janssen, R. A. J.; Cornil, J.; Brédas, J. L. Chem. —Eur. J. 1998, 4, 1509-1522.

(75) Rubio, M.; Merchan, M.; Orti, E. Chem. Phys. Chem. 2005, 6, 1357-1368.

(76) Rubio, M.; Merchan, M.; Orti, E.; Roos, B. J. Chem. Phys. 1995, $102,3580-3586$.

(77) Rubio, M.; Merchán, M.; Ortí, E.; Roos, B. O. Chem. Phys. Lett. 1996, 248, 321-328.

(78) Keszthelyi, T.; Grage, M. M.-L.; Offersgard, J. F.; Wilbrandt, R.; Svendsen, C.; Sonnich Mortensen, O.; Pedersen, J. K.; Jensen, H. J. A. J. Phys. Chem. A 2000, 104, 2808-2823. 
(79) Fichou, D.; Horowitz, G.; Garnier, F. Synth. Met. 1990, 39, 125-131.

(80) Rubio, M.; Ortí, E.; Pou-Amerigo, R.; Merchán, M. J. Phys. Chem. A 2001, 105, 9788-9794.

(81) Baer, R.; Livshits, E.; Salzner, U. Annu. Rev. Phys. Chem. 2010, 61, 85-109.

(82) Tsuneda, T.; Song, J.-W.; Suzuki, S.; Hirao, K. J. Chem. Phys. 2010, 133, 174101-9.

(83) Perdew, J. P.; Parr, R. G.; Levy, M.; Balduz, J. L. Phys. Rev. Lett. 1982, 49, 1691-1694.

(84) Almbladh, C. O.; Von Barth, U. Phys. Rev. B 1985, 31, 3231-3244.

(85) Holland, D. M. P.; Karlsson, L.; von Niessen, W. J. Electron Spectrosc. Relat. Phenom. 2001, 113, 221-239.

(86) Schiedt, J.; Knott, W. J.; Le Barbu, K.; Schlag, E. W.; Weinkauf, R. J. Chem. Phys. 2000, 113, 9470-9478.

(87) Djurovich, P. I.; Mayo, E. I.; Forrest, S. R.; Thompson, M. E. Org. Electronics 2009, 10, 515-520.

(88) Hill, I. G.; Kahn, A.; Soos, Z. G.; Pascal, J. R. A. Chem. Phys. Lett. 2000, 327, 181-188.

(89) Sato, N.; Seki, K.; Inokuchi, H. J. Chem. Soc., Faraday II 1981, 77, 1621-1633. 